MATHEMATICS OF COMPUTATION

Volume 74, Number 250, Pages 555-583

S 0025-5718(04)01720-X

Article electronically published on October 27, 2004

\title{
ALGORITHMS WITHOUT ACCURACY SATURATION FOR EVOLUTION EQUATIONS IN HILBERT AND BANACH SPACES
}

\author{
IVAN P. GAVRILYUK AND VOLODYMYR L. MAKAROV
}

\begin{abstract}
We consider the Cauchy problem for the first and the second order differential equations in Banach and Hilbert spaces with an operator coefficient $A(t)$ depending on the parameter $t$. We develop discretization methods with high parallelism level and without accuracy saturation; i.e., the accuracy adapts automatically to the smoothness of the solution. For analytical solutions the rate of convergence is exponential. These results can be viewed as a development of parallel approximations of the operator exponential $e^{-t A}$ and of the operator cosine family $\cos \sqrt{A} t$ with a constant operator $A$ possessing exponential accuracy and based on the Sinc-quadrature approximations of the corresponding Dunford-Cauchy integral representations of solutions or the solution operators.
\end{abstract}

\section{INTRODUCTION}

We consider the evolution problems

$$
\frac{d u}{d t}+A(t) u=f(t), \quad t \in(0, T] ; \quad u(0)=u_{0},
$$

and

$$
\frac{d^{2} u}{d t^{2}}+A(t) u=f(t), \quad t \in(0, T] ; \quad u(0)=u_{0}, \quad u^{\prime}(0)=u_{01}
$$

where $A(t)$ is a densely defined closed (unbounded) operator with the domain $D(A)$ independent of $t$ in a Banach space $X, u_{0}, u_{01}$ are given vectors and $f(t)$ is a given vector-valued function. We suppose the operator $A(t)$ to be strongly positive; i.e., there exists a positive constant $M_{R}$ independent of $t$ such that on the rays and outside a sector $\Sigma_{\theta}=\{z \in \mathbb{C}: 0 \leq \arg (z) \leq \theta, \theta \in(0, \pi / 2)\}$ the following resolvent estimate holds:

$$
\left\|(z I-A(t))^{-1}\right\| \leq \frac{M_{R}}{1+|z|} .
$$

This assumption implies that there exists a positive constant $c_{\kappa}$ such that (see [7], p. 103)

$$
\left\|A^{\kappa}(t) e^{-s A(t)}\right\| \leq c_{\kappa} s^{-\kappa}, \quad s>0, \quad \kappa \geq 0 .
$$

Our further assumption is that there exists a real positive $\omega$ such that

$$
\left\|e^{-s A(t)}\right\| \leq e^{-\omega s} \quad \forall s, t \in[0, T]
$$

Received by the editor January 21, 2003 and, in revised form, February 26, 2004.

2000 Mathematics Subject Classification. Primary 65J10, 65M70; Secondary 35K90, 35L90.

Key words and phrases. Evolution equation, parameter dependent operator, algorithms without accuracy saturation, exponentially convergent algorithms, Sinc-methods. 
(see [23], Corollary 3.8, p. 12, for corresponding assumptions on $A(t)$ ). Let us also assume that the conditions

$$
\begin{array}{cl}
\left\|[A(t)-A(s)] A^{-\gamma}(t)\right\| \leq \tilde{L}_{1, \gamma}|t-s| & \forall t, s, 0 \leq \gamma \leq 1, \\
\left\|A^{\beta}(t) A^{-\beta}(s)-I\right\| \leq \tilde{L}_{\beta}|t-s| & \forall t, s \in[0, T]
\end{array}
$$

hold. Note that efficient approximations without accuracy saturation or with the exponential accuracy for the solution operators of equations (1.1), (1.2) with an unbounded operator $A$ independent of $t$ were proposed in [8, 9, 13, 14]. Using the Cayley transform, one can represent the exact solution (or the solution operator) by a series. The truncated sum of $N$ series terms represents a numerical algorithm without accuracy saturation, i.e., with the convergence rate automatically adapting to the smoothness of the input data [8, 14]. Other approximations [9, 13] use representations of the exact solution or the solution operators through the resolvent of the spatial operator by the Dunford-Cauchy integral along a path in the complex plane enveloping the spectrum of the spatial operator. After parametrization this integral can be translated into an improper integral over the real axis and the last one is then approximated by a suitable Sinc-quadrature [26] possessing an exponential convergence rate. In this way we get an approximation to the solution operator as a short sum of resolvents of the spatial operator.

Sinc approximations can also be used to get exponentially convergent approximations to the solutions of various partial differential equations (PDE) with a known Green function or a fundamental solution which allow one to represent the solution by an integral [27]. Note that, in general, it is not the case for arbitrary equations of the type (1.1), (1.2) considered in the present paper.

The aim of this paper is to get algorithms without accuracy saturation and exponentially convergent algorithms for the solution of equations (1.1), (1.2). We use a piecewise constant approximation of the operator $A(t)$ and an exact integral corollary of these equations on the Chebyshev grid which is then approximated by the collocation method. The operator exponential (for equation (1.1)) and the operator cosine function (for equation (1.2)) with stationary operators involved in the algorithms can be computed by the Sinc approximations from [9, 13].

We begin with an example which shows the practical relevance for the assumptions above.

Example 1.1. Let $q(t) \geq q_{0}>0, t \in[0, T]$, be a given function from the Hölder class with the exponent $\alpha \in(0,1]$. We consider the operator $A(t)$ defined by

$$
\begin{aligned}
D(A(t)) & =\left\{u(x) \in H^{4}(0,1): u(0)=u^{\prime \prime}(0)=u(1)=u^{\prime \prime}(1)=0\right\}, \\
A(t) u & =\left[\frac{d^{2}}{d x^{2}}-q(t)\right]^{2} u=\frac{d^{4} u}{d x^{4}}-2 q(t) \frac{d^{2} u}{d x^{2}}+q^{2}(t) u \quad \forall u \in D(A(t))
\end{aligned}
$$

with the domain independent of $t$. It is easy to show that

$$
\begin{aligned}
D\left(A^{1 / 2}(t)\right) & =\left\{u(x) \in H^{2}(0,1): u(0)=u(1)=0\right\}, \\
A^{1 / 2}(t) & =-\frac{d^{2} u}{d x^{2}}+q(t) u \quad \forall u \in D\left(A^{1 / 2}(t)\right), \\
A^{-1 / 2}(t) & =\int_{0}^{1} G(x, \xi ; t) v(\xi) d \xi,
\end{aligned}
$$


where the Green function is given by

(1.10)

$$
G(x, \xi ; t)=\frac{1}{\sqrt{q(t)} \sinh \sqrt{q(t)}} \begin{cases}\sinh (\sqrt{q(t)} x) \sinh (\sqrt{q(t)}(1-\xi)), & \text { if } x \leq \xi, \\ \sinh (\sqrt{q(t)} \xi) \sinh (\sqrt{q(t)}(1-x)), & \text { if } \xi \leq x\end{cases}
$$

Then we have the relation

$$
\begin{aligned}
{[A(t)-A(s)] A^{-1 / 2}(t) v } & =[q(t)-q(s)]\left\{-2 \frac{d^{2}}{d x^{2}}+[q(t)+q(s)]\right\} \int_{0}^{1} G(x, \xi ; t) v(\xi) d \xi \\
& =[q(t)-q(s)]\left\{2 v(x)-[q(t)-q(s)] \int_{0}^{1} G(x, \xi ; t) v(\xi) d \xi\right\},
\end{aligned}
$$

which leads to the estimate

$$
\begin{aligned}
& \left\|[A(t)-A(s)] A^{-1 / 2}(t) v(\cdot)\right\|_{C[0,1] \rightarrow C[0,1]} \\
& \quad \leq L|t-s|^{\alpha}\left\{2\|v\|_{C[0,1]}+L|t-s|^{\alpha} \frac{1}{2 \sqrt{q(t)}} \tanh (\sqrt{q(t)} / 2)\|v\|_{C[0,1]}\right\},
\end{aligned}
$$

where $L$ is the Hölder constant. This inequality yields

$$
\begin{aligned}
& \left\|[A(t)-A(s)] A^{-1 / 2}(t)\right\|_{C[0,1] \rightarrow C[0,1]} \\
& \quad \leq L\left\{2+L T^{\alpha} \tanh (\sqrt{q(t)} / 2) /(2 \sqrt{q(t)})\right\}|t-s|^{\alpha} ;
\end{aligned}
$$

i.e., condition (1.6) is fulfilled with $\gamma=1 / 2$ provided that $\alpha=1$. Let us prove condition (1.7). We have

$$
\begin{aligned}
{\left[A^{1 / 2}(t) A^{-1 / 2}(s)-I\right] v } & =\left[-\frac{d^{2}}{d x^{2}}+q(t)\right] \int_{0}^{1} G(x, \xi ; s) v(\xi) d \xi-v(x) \\
& =[q(t)-q(s)] \int_{0}^{1} G(x, \xi ; s) v(\xi) d \xi
\end{aligned}
$$

from which it follows that

$$
\left\|A^{1 / 2}(t) A^{-1 / 2}(s)-I\right\|_{C[0,1] \rightarrow C[0,1]} \leq L \frac{\tanh (\sqrt{q(t)} / 2)}{2 \sqrt{q(t)}}|t-s|^{\alpha} ;
$$

i.e., condition (1.7) is fulfilled with $\beta=1 / 2, \delta=\alpha=1$.

Remark 1.2. It is clear that, in general, for elliptic operators inequalities (1.6) and (1.7) hold true with $\gamma=1, \beta=1$.

Remark 1.3. Assumption (4.1) is not restrictive due to stability results from [15. The two initial value problems

$$
\frac{d u}{d t}+A(t) u=f(t), \quad u(0)=u_{0}
$$

and

$$
\frac{d v}{d t}+B(t) v=g(t), \quad v(0)=v_{0}
$$

with densely defined, closed operators $A(t), B(t)$ having a common domain $D(A(t))$ $=D(B(t))$ independent of $t$ were considered. The following assumptions were made. 
(1) There exist bounded inverse operators $A^{-1}(t), B^{-1}(t)$ and for the resolvents $R_{A(t)}(z)=(z-A(t))^{-1}, R_{B(t)}(z)=(z-B(t))^{-1}$ we have

$$
\left\|R_{A(t)}(z)\right\| \leq \frac{1}{1+|z|},\left\|R_{B(t)}(z)\right\| \leq \frac{1}{1+|z|} \quad(\theta+\epsilon \leq|\arg z| \leq \pi)
$$

for all $\theta \in(0, \pi / 2), \epsilon>0$ uniformly in $t \in[0, T]$.

(2) The operators $A(t), B(t)$ are strongly differentiable on $D(A(t))=D(B(t))$.

(3) There exists a constant $M$ such that

$$
\left\|A^{\beta}(s) B^{-\beta}(s)\right\| \leq M .
$$

(4) For the evolution operators $U_{A}(t, s), U_{B}(t, s)$ we have

$$
\left\|A(t) U_{A}(t, s)\right\| \leq \frac{C}{t-s}, \quad\left\|B(t) U_{B}(t, s)\right\| \leq \frac{C}{t-s} .
$$

(5) There exist positive constants $C, C_{\beta}$ such that

$$
\left\|A^{\rho}(t) A^{-\rho}(s)-I\right\| \leq C|t-s|^{\alpha}
$$

and

$$
\left\|A^{\beta}(t) U_{A}(t, s)\right\| \leq \frac{C_{\beta}}{|t-s|^{\beta}},\left\|B^{\beta}(t) U_{B}(t, s)\right\| \leq \frac{C_{\beta}}{|t-s|^{\beta}}
$$

for $0 \leq \beta<\alpha+\beta$.

The following stability result for Banach spaces was proved in [15] under these assumptions:

$$
\begin{aligned}
\left\|A^{\beta}(t) z(t)\right\|= & \left\|A^{\beta}(t)(u(t)-v(t))\right\| \\
\leq & M\left\|A^{\beta}(0) z(0)\right\|+c_{\beta} M \max _{0 \leq s \leq T}\left\|[B(s)-A(s)] A^{-\beta}(s)\right\| \\
& \times \frac{t^{1-\beta}}{1-\beta}\left\{\left\|B^{\beta}(0) v(0)\right\|+\int_{0}^{t}\left\|B^{\beta}(s) g(s)\right\| d s\right\} \\
& +M \int_{0}^{t}\left\|B^{\beta}(s) g(s)\right\| d s .
\end{aligned}
$$

It is possible to avoid the restriction $\beta<1$ if we consider equations (1.16), (1.17) in a Hilbert space. In this case we assume that there exists an operator $C=C^{*} \geq c_{0} I$ such that

$$
\left\|[A(s)-B(s)] C^{-1}\right\| \leq \delta,
$$

$$
\begin{aligned}
& (A(s) y, C y) \geq c_{0}\|C y\|^{2}, \\
& (B(s) y, C y) \geq c_{0}\|C y\|^{2} \quad \forall s \in[0, T], c_{0}>0 .
\end{aligned}
$$


Then the following stability estimate is fulfilled [15]:

$$
\begin{aligned}
\frac{1}{2}(C z(t), z(t))+\left(c_{0}-\epsilon-\epsilon_{1}\right) \int_{0}^{t}\|C z(s)\|^{2} d s \\
\leq \max _{0 \leq s \leq T}\left\|[A(s)-B(s)] C^{-1}\right\|^{2} \frac{\left(c_{0}-\epsilon_{2}\right)^{-1}}{2 \epsilon} \\
\times\left[\frac{1}{4 \epsilon_{2}} \int_{0}^{t}\|g(s)\|^{2} d s+\frac{1}{2}\left(C v_{0}, v_{0}\right)\right] \\
+\frac{1}{2 \epsilon_{1}} \int_{0}^{t}\|f(s)-g(s)\|^{2} d s+\frac{1}{2}\left(C\left(u_{0}-v_{0}\right), u_{0}-v_{0}\right),
\end{aligned}
$$

with arbitrary positive numbers $\epsilon, \epsilon_{1}, \epsilon_{2}$ such that $\epsilon+\epsilon_{1}<c_{0}, \epsilon_{2}<c_{0}$ which stand for the stability with respect to the right-hand side, the initial condition and the coefficient stability. Note that an analogous estimate in the case of a finite dimensional Hilbert spaces and of a constant operator $A$ was proved in [25, p. 62].

Example 1.4. Let $\Omega \subset \mathbb{R}^{2}$ be a polygon and let

$$
\mathcal{L}(x, t, D)=-\sum_{i, j=1}^{2} \frac{\partial}{\partial x_{i}} a_{i, j}(x, t) \frac{\partial}{\partial x_{j}}+\sum_{j=1}^{2} b_{j}(x, t) \frac{\partial}{\partial x_{j}}+c(x, t)
$$

be a second order elliptic operator with time-dependent real smooth coefficients satisfying the uniform ellipticity condition

$$
\sum_{i, j=1}^{2} a_{i j}(x, t) \xi_{i} \xi_{j} \geq \delta_{1}|\xi|^{2} \quad\left(\xi=\left(\xi_{1}, \xi_{2}\right) \in \mathbb{R}\right)
$$

with a positive constant $\delta_{1}$. Taking $X=L^{2}(\Omega)$ and $V=H_{0}^{1}(\Omega)$ or $V=H^{1}(\Omega)$ accordingly to the boundary condition

$$
u=0 \quad \text { on } \quad \partial \Omega \times(0, T)
$$

or

$$
\frac{\partial u}{\partial \nu_{\mathcal{L}}}+\sigma u=0 \quad \text { on } \quad \partial \Omega \times(0, T)
$$

we set

$$
\begin{aligned}
\mathcal{A}_{t}(u, v)= & \sum_{i, j=1}^{2} \int_{\Omega} a_{i, j}(x, t) \frac{\partial u}{\partial x_{i}} \frac{\partial v}{\partial x_{j}} d x \\
& +\sum_{j=1}^{2} \int_{\Omega} b_{j}(x, t) \frac{\partial u}{\partial x_{j}} v d x+\int_{\Omega} c(x, t) u v d x+\int_{\partial \Omega} \sigma(x, t) u v d S
\end{aligned}
$$

for $u, v \in V$. An $m$-sectorial operator $A(t)$ in $X$ can be defined through the relation

$$
\mathcal{A}_{t}(u, v)=(A(t) u, v)
$$

where $u \in D(A(t)) \subset V$ and $v \in V$. The relation

$$
D(A(t))=H^{2}(\Omega) \cap H_{0}^{1}(\Omega)
$$

follows for $V=H_{0}^{1}(\Omega)$ and

$$
D(A(t))=\left\{v \in H^{2}(\Omega) \mid \frac{\partial v}{\partial \nu_{\mathcal{L}}} \text { on } \partial \Omega\right\}
$$

for $V=H^{1}(\Omega)$, if $\partial \Omega$ is smooth for instance. 
It was proven in [7, pp. 95-101] that all the assumptions above hold for such an operator $A(t)$.

As we will see below, the parameter $\gamma$ from (1.6) plays an essential role for the construction and the analysis of discrete approximations and algorithms for problems (1.1), (1.2).

\section{Discrete First order PROBlem IN The CASE $\gamma<1$}

For the sake of simplicity we consider problem (1.1) on the interval $[-1,1]$ (if it is not the case, one can reduce problem (1.1) to this interval by the variable transform $\left.t=2 t^{\prime} / T-1, t \in[-1,1], t^{\prime} \in[0, T]\right)$. We choose a mesh $\omega_{n}$ of $n$ various points $\omega_{n}=\left\{t_{k}=\cos \frac{(2 k-1) \pi}{2 n}, k=1, \ldots, n\right\}$ on $[-1,1]$ and set $\tau_{k}=t_{k}-t_{k-1}$,

$$
\bar{A}(t)=A_{k}=A\left(t_{k}\right), \quad t \in\left(t_{k-1}, t_{k}\right],
$$

where $t_{k}$ are zeros of Chebyshev orthogonal polynomial of the first kind $T_{n}(t)=$ $\cos (n \arccos t)$. Let $t_{\nu}=\cos \theta_{\nu}, 0<\theta_{\nu}<\pi, \nu=1,2, \ldots, n$, be zeros of the Chebyshev orthogonal polynomial $T_{n}(t)$ taken in decreasing order. Then it is well known that (see [28], Ch.6, Th.6.11.12, 29], p. 123)

$$
\begin{aligned}
t_{\nu+1}-t_{\nu} & <\frac{\pi}{n}, \quad \nu=1, \ldots, n, \\
\tau_{\max } & =\max _{1 \leq k \leq n} \tau_{k}<\frac{\pi}{n} .
\end{aligned}
$$

Let us rewrite problem (1.1) in the form

$$
\begin{aligned}
\frac{d u}{d t}+\bar{A}(t) u= & {[\bar{A}(t)-A(t)] u(t)+f(t), } \\
& u(0)=u_{0}
\end{aligned}
$$

from which we deduce

$$
\begin{aligned}
u(t)= & e^{-A_{k}\left(t-t_{k-1}\right)} u\left(t_{k-1}\right) \\
& +\int_{t_{k-1}}^{t} e^{-A_{k}(t-\eta)}\left\{\left[A_{k}-A(\eta)\right] u(\eta)+f(\eta)\right\} d \eta, \quad t \in\left[t_{k-1}, t_{k}\right] .
\end{aligned}
$$

Since $A_{k-1}$ and $e^{-A_{k-1} \tau_{k}}$ commute, assumption (1.7) yields

$$
\left\|A_{k}^{\beta} A_{p}^{-\beta}\right\| \leq 1+\left\|A_{k}^{\beta} A_{p}^{-\beta}-I\right\| \leq 1+\tilde{L}_{\beta}\left|t_{k}-t_{p}\right| \leq 1+\tilde{L}_{\beta} T .
$$

Let

$$
P_{n-1}(t ; u)=P_{n-1} u=\sum_{p=1}^{n} u\left(t_{p}\right) L_{p, n-1}(t)
$$

be the interpolation polynomial for the function $u(t)$ on the mesh $\omega_{n}$, let $y=$ $\left(y_{1}, \ldots, y_{n}\right), y_{i} \in X$ be a given vector, and let

$$
P_{n-1}(t ; y)=P_{n-1} y=\sum_{p=1}^{n} y_{p} L_{p, n-1}(t)
$$

be the polynomial that interpolates $y$, where $L_{p, n-1}=\frac{T_{n}(t)}{T_{n}^{\prime}\left(t_{p}\right)\left(t-t_{p}\right)}, p=1, \ldots, n$, are the Lagrange fundamental polynomials. 
Substituting $P_{n}(\eta ; y)$ for $u(\eta)$ and $y_{k}$ for $u\left(t_{k}\right)$ in (2.4), we arrive at the following system of linear equations with respect to the unknowns $y_{k}$ :

$$
y_{k}=e^{-A_{k} \tau_{k}} y_{k-1}+\sum_{p=0}^{n} \alpha_{k p} y_{p}+\phi_{k}, \quad k=1, \ldots, n,
$$

where

$$
\begin{gathered}
\alpha_{k p}=\int_{t_{k-1}}^{t_{k}} e^{-A_{k}\left(t_{k}-\eta\right)}\left[A_{k}-A(\eta)\right] L_{p, n-1}(\eta) d \eta, \\
\phi_{k}=\int_{t_{k-1}}^{t_{k}} e^{-A_{k}\left(t_{k}-\eta\right)} f(\eta) d \eta .
\end{gathered}
$$

Remark 2.1. In order to compute $\alpha_{k p}$ and $\phi_{k}$ efficiently, we replace $A(t), f(t)$ by their interpolation polynomials (it is possible due to stability results (1.23), (1.26); see also [15]) and then calculate the integrals analytically. We have

$$
\begin{gathered}
A(t)=\sum_{l=1}^{n} \frac{A_{l}}{t-t_{l}} \frac{T_{n}(t)}{T_{n}^{\prime}\left(t_{l}\right)}, \\
f(t)=\sum_{l=1}^{n} \frac{f_{l}}{t-t_{l}} \frac{T_{n}(t)}{T_{n}^{\prime}\left(t_{l}\right)}, \quad f_{l}=f\left(t_{l}\right),
\end{gathered}
$$

so that

$$
\begin{gathered}
\alpha_{k p}=\frac{1}{T_{n}^{\prime}\left(t_{p}\right)} \sum_{l=1}^{n} \frac{1}{T_{n}^{\prime}\left(t_{l}\right)} \int_{t_{k-1}}^{t_{k}} e^{-A_{k}\left(t_{k}-\eta\right)} \frac{T_{n}^{2}(\eta)}{\left(\eta-t_{l}\right)\left(\eta-t_{p}\right)} d \eta\left[A_{k}-A_{l}\right] \\
\phi_{k}=\sum_{l=1}^{n} \frac{f_{k}}{T_{n}^{\prime}\left(t_{l}\right)} \int_{t_{k-1}}^{t_{k}} e^{-A_{k}\left(t_{k}-\eta\right)} \frac{T_{n}(\eta)}{\eta-t_{l}} d \eta .
\end{gathered}
$$

Using the relation $2 T_{n}^{2}(\eta)=1+2 T_{2 n}(\eta)$, the polynomial $p_{2 n-2}^{(l, p)}=\frac{T_{n}^{2}(\eta)}{\left(\eta-t_{l}\right)\left(\eta-t_{p}\right)}$ can be represented as (see [2])

$$
\begin{aligned}
p_{2 n-2}^{(l, p)} & =\frac{2 T_{2 n}(\eta)+1}{2\left(\eta-t_{l}\right)\left(\eta-t_{p}\right)} \\
& =\frac{1}{2\left(\eta-t_{l}\right)\left(\eta-t_{p}\right)}\left[2 n \sum_{m=0}^{n} \frac{(-1)^{m}(2 n-m-1) !}{m !(2 n-2 m) !}(2 \eta)^{2 n-2 m}+1\right] \\
& =\sum_{i=0}^{2 n-2} q_{i}(l, p) \eta^{2 n-2-i},
\end{aligned}
$$

where the coefficients $q_{i}(l, p)$ can be calculated, for example, by the Horner scheme. Given $q_{i}(l, p)$, we furthermore find that

$$
\alpha_{k p}=\frac{1}{T_{n}^{\prime}\left(t_{p}\right)} \sum_{l=1}^{n} \frac{1}{T_{n}^{\prime}\left(t_{l}\right)} \sum_{i=0}^{2 n-2} q_{i}(l, p) I_{k, i}\left[A_{k}-A_{l}\right],
$$


where

$$
\begin{aligned}
I_{k, i}= & \int_{t_{k-1}}^{t_{k}} e^{-A_{k}\left(t_{k}-\eta\right)} \eta^{2 n-2-i} d \eta \\
= & \sum_{s=0}^{2 n-2-i}(-1)^{s}(2 n-2-i)(2 n-3-i) \\
& \cdots(2 n-2-i-s+1) A_{k}^{-s-1} t_{k}^{2 n-2-i-s} \\
& -\sum_{s=0}^{2 n-2-i}(-1)^{s}(2 n-2-i)(2 n-3-i) \\
& \cdots(2 n-2-i-s+1) A_{k}^{-s-1} t_{k-1}^{2 n-2-i-s} e^{-A_{k} \tau_{k}} .
\end{aligned}
$$

Analogously one can also calculate $\phi_{k}$.

For the error $z=\left(z_{0}, z_{1}, \ldots, z_{n}\right), z_{k}=u\left(t_{k}\right)-y_{k}$ we have the relations

$$
z_{k}=e^{-A_{k} \tau_{k}} z_{k-1}+\sum_{p=0}^{n} \alpha_{k p} z_{p}+\psi_{k}, \quad k=1, \ldots, n,
$$

where

$$
\psi_{k}=\int_{t_{k-1}}^{t_{k}} e^{-A_{k}\left(t_{k}-\eta\right)}\left[A_{k}-A(\eta)\right]\left[u(\eta)-P_{n}(\eta ; u)\right] d \eta .
$$

We introduce the matrix

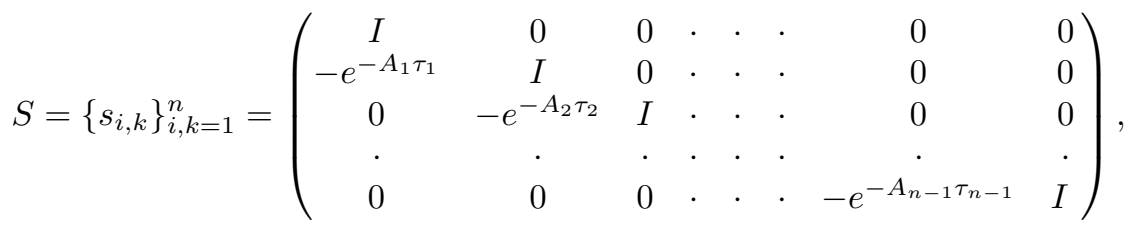

the matrix $C=\left\{\tilde{\alpha}_{k, p}\right\}_{k, p=1}^{n}$ with $\tilde{\alpha}_{k, p}=A_{k}^{\gamma} \alpha_{k, p} A_{p}^{-\gamma}$ and the vectors

$$
y=\left(\begin{array}{c}
A_{1}^{\gamma} y_{1} \\
\cdot \\
\cdot \\
\cdot \\
A_{n}^{\gamma} y_{n}
\end{array}\right), f=\left(\begin{array}{c}
A_{1}^{\gamma} \phi_{1} \\
\cdot \\
\cdot \\
\cdot \\
a_{n}^{\gamma} \phi_{n}
\end{array}\right), \tilde{f}=\left(\begin{array}{c}
A_{1}^{\gamma} e^{-A_{1} \tau_{1}} u_{0} \\
0 \\
\cdot \\
\cdot \\
\cdot \\
0
\end{array}\right) \psi=\left(\begin{array}{c}
A_{1}^{\gamma} \psi_{1} \\
\cdot \\
\cdot \\
\cdot \\
A_{n}^{\gamma} \psi_{n}
\end{array}\right) .
$$

It is easy to see that for

$$
\begin{aligned}
& S^{-1}=\left\{s_{i, k}^{-1}\right\}_{i, k=1}^{n} \\
& =\left(\begin{array}{ccccc}
I & 0 & \cdots & 0 & 0 \\
e^{-A_{1} \tau_{1}} & I & \cdots & 0 & 0 \\
e^{-A_{2} \tau_{2}} e^{-A_{1} \tau_{1}} & e^{-A_{2} \tau_{2}} & \cdots & 0 & 0 \\
\cdot & \cdot & \cdots & \cdot & \cdot \\
e^{-A_{n-1} \tau_{n-1}} \cdots e^{-A_{1} \tau_{1}} & e^{-A_{n-1} \tau_{n-1}} \cdots e^{-A_{2} \tau_{2}} & \cdots & e^{-A_{n-1} \tau_{n-1}} & I
\end{array}\right)
\end{aligned}
$$

we have

$$
S^{-1} S=\left(\begin{array}{cccc}
I & 0 & \cdots & 0 \\
0 & I & \cdots & 0 \\
. & . & \cdots & . \\
0 & 0 & \cdots & I
\end{array}\right)
$$


Remark 2.2. Using results of [9] 13, one can get a parallel and sparse approximation with an exponential convergence rate of operator exponentials in $S^{-1}$ and as a consequence a parallel and sparse approximation of $S^{-1}$.

We get from (2.8, 2.15)

$$
\begin{gathered}
A_{k}^{\gamma} y_{k}=e^{-A_{k} \tau_{k}} A_{k}^{\gamma} y_{k-1}+\sum_{p=0}^{n} \tilde{\alpha}_{k p} A_{p}^{\gamma} y_{p}+A_{k}^{\gamma} \phi_{k}, \\
A_{k}^{\gamma} z_{k}=e^{-A_{k} \tau_{k}} A_{k}^{\gamma} z_{k-1}+\sum_{p=0}^{n} \tilde{\alpha}_{k p} A_{p}^{\gamma} z_{p}+A_{k}^{\gamma} \psi_{k}, \quad k=1, \ldots, n,
\end{gathered}
$$

or in matrix form

$$
\begin{gathered}
S y=C y+f-\tilde{f}, \\
S z=C z+\psi
\end{gathered}
$$

with

$$
z=\left(\begin{array}{c}
A_{1}^{\gamma} z_{1} \\
\cdot \\
\cdot \\
\cdot \\
A_{n}^{\gamma} z_{n}
\end{array}\right)
$$

Next, for a vector $v=\left(v_{1}, v_{2}, \ldots, v_{n}\right)^{T}$ and a block operator matrix $A=$ $\left\{a_{i j}\right\}_{i, j=1}^{n}$ we introduce the vector norm

$$
|\|v\|| \equiv|\|v\||_{\infty}=\max _{1 \leq k \leq n}\left\|v_{k}\right\|
$$

and the consistent matrix norm

$$
|\|A\|| \equiv|\|A\||_{\infty}=\max _{1 \leq i \leq n} \sum_{j=1}^{n}\left\|a_{i, j}\right\| .
$$

Due to (1.5) we get

$$
|| S^{-1} \| \mid \leq n .
$$

For further analysis we need the following auxiliary result.

Lemma 2.3. The estimates

$$
\begin{gathered}
|\| C||| \leq c\left(1+\tilde{L}_{\gamma} T\right) n^{\gamma-2} \ln n, \\
\left|\left\|S^{-1} C\right\|\right| \leq c\left(1+\tilde{L}_{\gamma} T\right) n^{\gamma-1} \ln n
\end{gathered}
$$

with a positive constant $c$ independent of $n$ hold true.

Proof. Assumption (1.6) together with (2.5) implies

$$
\begin{aligned}
\left\|\tilde{\alpha}_{k p}\right\| & =\left\|A_{k}^{\gamma} \alpha_{k p} A_{p}^{-\gamma}\right\| \\
& =\left\|\int_{t_{k-1}}^{t_{k}} A_{k}^{\gamma} e^{-A_{k}\left(t_{k}-\eta\right)}\left[A_{k}-A(\eta)\right] A_{p}^{-\gamma} L_{p, n-1}(\eta) d \eta\right\| \\
& \leq\left(1+\tilde{L}_{\gamma} T\right) \tau_{\max }^{1-\gamma} \int_{t_{k-1}}^{t_{k}}\left|L_{p, n-1}(\eta)\right| d \eta, \quad T=2 .
\end{aligned}
$$


Using the well-known estimate for the Lebesgue constant $\Lambda_{n}$ related to the Chebyshev interpolation nodes (see, e.g., [28, 29])

$$
\Lambda_{n}=\max _{\eta \in[-1,1]} \sum_{p=1}^{n}\left|L_{p, n-1}(\eta)\right| \leq c \ln n
$$

and (2.29), we have

$$
\begin{aligned}
\|\| C \| & \leq \max _{1 \leq k \leq n} \sum_{p=1}^{n}\left\|\tilde{\alpha}_{k p}\right\| \\
& \leq\left(1+\tilde{L}_{\gamma} T\right) \tau_{\max }^{2-\gamma} \max _{\eta \in[-1,1]} \sum_{p=1}^{n}\left|L_{p, n-1}(\eta)\right| \\
& \leq\left(1+\tilde{L}_{\gamma} T\right) \tau_{\max }^{2-\gamma} \Lambda_{n} \leq c\left(1+\tilde{L}_{\gamma} T\right) \tau_{\max }^{2-\gamma} \ln n \\
& \leq c\left(1+\tilde{L}_{\gamma} T\right) n^{\gamma-2} \ln n
\end{aligned}
$$

with some positive constant $c$ independent of $n$. This estimate together with (2.26) implies

$$
\text { | }\left\|S^{-1} C\right\| \mid \leq c\left(1+\tilde{L}_{\gamma} T\right) n^{\gamma-1} \ln n \rightarrow 0
$$

as $n \rightarrow \infty$ provided that $\gamma<1$.

Remark 2.4. We have reduced the interval length to $T=2$ but we write $T$ explicitly in order to underline the dependence of constants involved on $T$ in the general case.

Let $\Pi_{n-1}$ be the set of all polynomials in $t$ with vector coefficients of degree less than or equal to $n-1$. Then the Lebesgue inequality

$$
\begin{aligned}
& \left\|u(\eta)-P_{n-1}(\eta ; u)\right\|_{C[-1,1]} \\
& \quad \equiv \max _{\eta \in[-1,1]}\left\|u(\eta)-P_{n-1}(\eta ; u)\right\| \leq\left(1+\Lambda_{n}\right) E_{n}(u)
\end{aligned}
$$

can be proved for vector-valued functions in complete analogy with [1, 28, 29] and with the error of the best approximation of $u$ by polynomials of degree not greater than $n-1$

$$
E_{n}(u)=\inf _{p \in \Pi_{n-1}} \max _{\eta \in[-1,1]}\|u(\eta)-p(\eta)\| .
$$

Now, we can go over to the main result of this section.

Theorem 2.5. Let assumptions (1.3)-(1.7) with $\gamma<1$ hold. Then there exists a positive constant $c$ such that the following hold.

(1) For $n$ large enough it holds that

$$
|\|z\|| \equiv|\|y-u\|| \leq c n^{\gamma-1} \ln n E_{n}\left(A_{0}^{\gamma} u\right),
$$

where $u$ is the solution of (1.1).

(2) The system of linear algebraic equations

$$
S y=C y+f
$$

with respect to the approximate solution y can be solved by the fixed-point iteration

$$
y^{(k+1)}=S^{-1} C y^{(k)}+S^{-1}(f-\tilde{f}), \quad k=0,1, \ldots ; \quad y^{(0)} \text { arbitrary }
$$


with the convergence rate of a geometrical progression with the denominator $q \leq c n^{\gamma-1} \ln n<1$ for $n$ large enough.

Proof. From the second equation in $(2.22)$ we get

$$
z=S^{-1} C z+S^{-1} \psi
$$

from which due to Lemma 2.3 and $(2.26)$ we get

$$
|\|z\|| \leq c n|\|\psi\||
$$

for $n$ large enough. The last norm can be estimated in the following way:

$$
\begin{aligned}
&\|\| \psi \| \mid= \max _{1 \leq k \leq n} \| \int_{t_{k-1}}^{t_{k}}\left[A_{k}^{\gamma} e^{-A_{k}\left(t_{k}-\eta\right)}\left[A_{k}-A(\eta)\right]\right. \\
&\left.\times A_{k}^{-\gamma}\left(A_{k}^{\gamma} A_{0}^{-\gamma}\right)\left(A_{0}^{\gamma} u(\eta)-P_{n}\left(\eta ; A_{0}^{\gamma} u\right)\right)\right] d \eta \| \\
& \leq\left(1+\tilde{L}_{\gamma} T\right) \max _{1 \leq k \leq n} \int_{t_{k-1}}^{t_{k}}\left|t_{k}-\eta\right|^{-\gamma}\left|t_{k}-\eta\right| \\
& \times\left\|A_{0}^{\gamma} u(\eta)-P_{n}\left(\eta ; A_{0}^{\gamma} u\right)\right\| d \eta \\
& \leq\left(1+\tilde{L}_{\gamma} T\right) \tau_{\max }^{2-\gamma}\left\|A_{0}^{\gamma} u(\cdot)-P_{n-1}\left(\cdot ; A_{0}^{\gamma} u\right)\right\|_{C[-1,1]} \\
& \leq\left(1+\tilde{L}_{\gamma} T\right) \tau_{\max }^{2-\gamma}\left(1+\Lambda_{n}\right) E_{n}\left(A_{0}^{\gamma} u\right) \leq c n^{\gamma-2} \ln n E_{n}\left(A_{0}^{\gamma} u\right),
\end{aligned}
$$

and taking into account (2.39), we get the statement of the theorem.

\section{DiscRete First ORDER PROBLEM IN THE CASE $\gamma \leq 1$}

In this section we construct a new discrete approximation of problem (1.1) which is a little more complicated than approximation (2.8) of the previous section but possesses a higher convergence order and allows the case $\gamma=1$.

Applying transform (2.4) once more (i.e., substituting $u(t)$ recursively), we get

$$
\begin{aligned}
u(t)= & \left\{e^{-A_{k}\left(t-t_{k-1}\right)}+\int_{t_{k-1}}^{t} e^{-A_{k}(t-\eta)}\left[A_{k}-A(\eta)\right] e^{-A_{k}\left(\eta-t_{k-1}\right)} d \eta\right\} u\left(t_{k-1}\right) \\
& +\int_{t_{k-1}}^{t} e^{-A_{k}(t-\eta)}\left[A_{k}-A(\eta)\right] \int_{t_{k-1}}^{\eta} e^{-A_{k}(\eta-s)}\left[A_{k}-A(s)\right] u(s) d s d \eta \\
& +\int_{t_{k-1}}^{t} e^{-A_{k}(t-\eta)}\left\{\left[A_{k}-A(\eta)\right] \int_{t_{k-1}}^{\eta} e^{-A_{k}(\eta-s)} f(s) d s+f(\eta)\right\} d \eta
\end{aligned}
$$

Setting $t=t_{k}$, we arrive at the relation

$$
\begin{aligned}
u\left(t_{k}\right)= & S_{k, k-1} u\left(t_{k-1}\right) \\
& +\int_{t_{k-1}}^{t_{k}} e^{-A_{k}\left(t_{k}-\eta\right)}\left[A_{k}-A(\eta)\right] \int_{t_{k-1}}^{\eta} e^{-A_{k}(\eta-s)}\left[A_{k}-A(s)\right] u(s) d s d \eta+\phi_{k},
\end{aligned}
$$


where

$$
\begin{aligned}
S_{k, k-1}= & e^{-A_{k} \tau_{k}}+\int_{t_{k-1}}^{t_{k}} e^{-A_{k}\left(t_{k}-\eta\right)}\left[A_{k}-A(\eta)\right] e^{-A_{k}\left(\eta-t_{k-1}\right)} d \eta, \\
\phi_{k}= & \int_{t_{k-1}}^{t_{k}} e^{-A_{k}\left(t_{k}-\eta\right)} f(\eta) d \eta \\
& +\int_{t_{k-1}}^{t_{k}} e^{-A_{k}\left(t_{k}-\eta\right)}\left[A_{k}-A(\eta)\right] \int_{t_{k-1}}^{\eta} e^{-A_{k}(\eta-s)} f(s) d s d \eta .
\end{aligned}
$$

Substituting the interpolation polynomial $P_{n-1}(\eta ; y)$ from the previous section for $u(\eta)$ and $y_{k}$ for $u\left(t_{k}\right)$ in (3.2), we arrive at the following system of linear equations with respect to the unknowns $y_{k}$ :

$$
y_{k}=S_{k, k-1} y_{k-1}+\sum_{p=1}^{n} \alpha_{k p} y_{p}+\phi_{k}, \quad k=1, \ldots, n,
$$

where

$$
\begin{aligned}
\alpha_{k p}= & \int_{t_{k-1}}^{t_{k}} e^{-A_{k}\left(t_{k}-\eta\right)}\left[A_{k}-A(\eta)\right] \\
& \times \int_{t_{k-1}}^{\eta} e^{-A_{k}(\eta-s)}\left[A_{k}-A(s)\right] L_{p, n-1}(s) d s d \eta .
\end{aligned}
$$

Remark 3.1. Due to stability results (1.23), (1.26) (see also [15]) one can approximate the initial problems by problems with polynomials $\tilde{A}(t), \tilde{f}(t)$, for example, as interpolation polynomials for $A(t), f(t)$.

With the aim of getting a computational algorithm for $\alpha_{k p}$, we write down formula (3.5) in the form

$$
\begin{aligned}
\alpha_{k p}= & \int_{t_{k-1}}^{t_{k}} \int_{t_{k-1}}^{\eta} e^{-A_{k}\left(t_{k}-\eta\right)} \\
& \times\left[A_{k}-A(\eta)\right] e^{-A_{k}(\eta-s)} d \eta\left[A_{k}-A(s)\right] L_{p, n-1}(s) d s .
\end{aligned}
$$

In order to calculate the inner integral, we represent

$$
A(\eta)=A_{k}+\left(t_{k}-\eta\right) B_{1, k}+\cdots+\left(t_{k}-\eta\right)^{n-1} B_{n-1, k} .
$$

Then we have to calculate integrals of the type

$$
\tilde{\alpha}_{k p}=\int_{t_{k-1}}^{\eta} e^{-A_{k}\left(t_{k}-\eta\right)}\left(t_{k}-\eta\right)^{p} B_{p, k} e^{-A_{k}(\eta-s)} d \eta .
$$

Analogously to [15, using the representation by the Dunford-Cauchy integrals and the residue theorem under assumption of the strong P-positiveness [8, 13, 9] of the operator $A(t)$, one can get

$$
\tilde{\alpha}_{k p}=\frac{p !}{2 \pi i} \int_{\Gamma_{I}} e^{-z\left(t_{k}-\eta\right)}\left(A_{k}-z I\right)^{-p-1} B_{p, k}\left(z I-A_{k}\right)^{-1} d z,
$$

where $\Gamma_{I}$ is an integration parabola enveloping the spectral parabola of the strongly P-positive operator $A(t)$. Now, using (3.7), (3.9) , formula (3.6) can be written down 
as

$$
\begin{aligned}
\alpha_{k p}= & -\frac{1}{2 \pi i} \int_{\Gamma_{I}} \sum_{p=1}^{n-1} p !\left(A_{k}-z I\right)^{-p-1} B_{p, k}\left(z I-A_{k}\right)^{-1} \\
& \times \int_{t_{k-1}}^{t_{k}} e^{-z\left(t_{k}-s\right)}\left[A_{k}-A(s)\right] L_{p, n-1}(s) d s d z
\end{aligned}
$$

The inner integral in this formula can be calculated analogously to (2.13), and the integral along $\Gamma_{I}$ can be calculated explicitly using the residue theorem.

For the error $z=\left(z_{1}, \ldots, z_{n}\right), z_{k}=u\left(t_{k}\right)-y_{k}$ we have the relations

$$
z_{k}=S_{k, k-1} z_{k-1}+\sum_{p=0}^{n} \alpha_{k p} z_{p}+\psi_{k}, \quad k=1, \ldots, n,
$$

where

$$
\psi_{k}=\int_{t_{k-1}}^{t_{k}} e^{-A_{k}\left(t_{k}-\eta\right)} \int_{t_{k-1}}^{\eta} e^{-A_{k}(\eta-s)}\left[A_{k}-A(s)\right]\left[u(s)-P_{n-1}(s ; u)\right] d s d \eta .
$$

We introduce the matrix

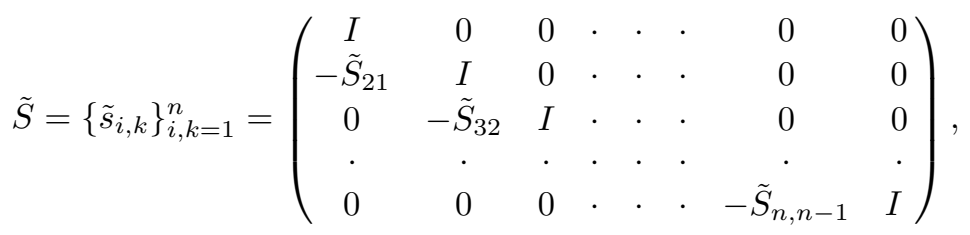

with $\tilde{S}_{k, k-1}=A_{k}^{\gamma} S_{k, k-1} A_{k-1}^{-\gamma}$, the matrix $C=\left\{\tilde{\alpha}_{k, p}\right\}_{k, p=1}^{n}$ with $\tilde{\alpha}_{k, p}=A_{k}^{\gamma} \alpha_{k, p} A_{p}^{-\gamma}$ and the vectors

$$
y=\left(\begin{array}{c}
A_{1}^{\gamma} y_{1} \\
\cdot \\
\cdot \\
\cdot \\
A_{n}^{\gamma} y_{n}
\end{array}\right), f=\left(\begin{array}{c}
A_{1}^{\gamma} \phi_{1} \\
\cdot \\
\cdot \\
\cdot \\
a_{n}^{\gamma} \phi_{n}
\end{array}\right), \psi=\left(\begin{array}{c}
A_{1}^{\gamma} \psi_{1} \\
\cdot \\
\cdot \\
\cdot \\
A_{n}^{\gamma} \psi_{n}
\end{array}\right), \tilde{f}=\left(\begin{array}{c}
A_{1}^{\gamma} S_{21} u_{0} \\
0 \\
\cdot \\
\cdot \\
\cdot \\
0
\end{array}\right) .
$$

It is easy to check that for

$$
\begin{aligned}
& \tilde{S}^{-1}=\left\{\tilde{s}_{i, k}^{-1}\right\}_{i, k=1}^{n} \\
& =\left(\begin{array}{cccccc}
I & 0 & 0 & \cdots & 0 & 0 \\
\tilde{S}_{21} & I & 0 & \cdots & 0 & 0 \\
\tilde{S}_{32} \tilde{S}_{21} & \tilde{S}_{32} & I & \cdots & 0 & 0 \\
\cdot & \cdot & \cdot & \cdots & \cdot & \cdot \\
\tilde{S}_{n, n-1} \cdots \tilde{S}_{21} & \tilde{S}_{n, n-1} \cdots \tilde{S}_{32} & \tilde{S}_{n, n-1} \cdots \tilde{S}_{43} & \cdots & \tilde{S}_{n, n-1} & I
\end{array}\right)
\end{aligned}
$$

we have that

$$
\tilde{S}^{-1} \tilde{S}=\left(\begin{array}{cccc}
I & 0 & \cdots & 0 \\
0 & I & \cdots & 0 \\
. & . & \cdots & . \\
0 & 0 & \cdots & I
\end{array}\right)
$$


Remark 3.2. Using results of 9], one can get a parallel and sparse approximation of operator exponentials in $\tilde{S}^{-1}$ and as a consequence a parallel and sparse approximation of $\tilde{S}^{-1}$.

We get from (3.4), (3.11)

$$
\begin{aligned}
& A_{k}^{\gamma} y_{k}=\tilde{S}_{k, k-1} A_{k-1}^{\gamma} y_{k-1}+\sum_{p=0}^{n} \tilde{\alpha}_{k p} A_{p}^{\gamma} y_{p}+A_{k}^{\gamma} \phi_{k}, \\
& A_{k}^{\gamma} z_{k}=\tilde{S}_{k, k-1} A_{k-1}^{\gamma} z_{k-1}+\sum_{p=0}^{n} \tilde{\alpha}_{k p} A_{p}^{\gamma} z_{p}+A_{k}^{\gamma} \psi_{k},
\end{aligned}
$$

or in matrix form

$$
\begin{gathered}
\tilde{S} y=C y+f+\tilde{f}, \\
\tilde{S} z=C z+\psi
\end{gathered}
$$

with

$$
z=\left(\begin{array}{c}
A_{1}^{\gamma} z_{1} \\
\cdot \\
\cdot \\
\cdot \\
A_{n}^{\gamma} z_{n}
\end{array}\right) .
$$

In the next lemma we estimate the norms of $C$ and $\tilde{S}^{-1} C$.

Lemma 3.3. The estimates

$$
\begin{gathered}
|\|C\|| \leq c(\gamma, T) n^{2 \gamma-4} \ln n, \\
\left|\left\|\tilde{S}^{-1} C\right\|\right| \leq c(\gamma, T) n^{2 \gamma-3} \ln n
\end{gathered}
$$

with a positive constant $c=c(T, \gamma)$ depending on $\gamma$ and the interval length $T$ but independent of $n$ and such that $c=c(T, \gamma) \rightarrow \infty$ as $\gamma \rightarrow 1$ hold true.

Proof. Assumption (1.6) together with (1.4), (2.5), (2.2), (2.30) imply

$$
\begin{aligned}
\left\|\tilde{\alpha}_{k p}\right\|= & \left\|A_{k}^{\gamma} \alpha_{k p} A_{p}^{-\gamma}\right\| \\
= & \| \int_{t_{k-1}}^{t_{k}} A_{k}^{\gamma} e^{-A_{k}\left(t_{k}-\eta\right)}\left[A_{k}-A(\eta)\right] \\
& \times A_{k}^{-\gamma} \int_{t_{k-1}}^{\eta} A_{k}^{\gamma} e^{-A_{k}(\eta-s)}\left[A_{k}-A(s)\right] A_{p}^{-\gamma} L_{p, n-1}(\eta) d \eta \| \\
\leq & \left(1+\tilde{L}_{\gamma} T\right)\left(c_{\gamma} \tilde{L}_{1, \gamma}\right)^{2} \int_{t_{k-1}}^{t_{k}}\left|t_{k}-\eta\right|^{1-\gamma} \int_{t_{k-1}}^{\eta}|\eta-s|^{-\gamma}\left|t_{k}-s \| L_{p, n-1}(s)\right| d s d \eta .
\end{aligned}
$$


Due to (3.22) we have

$$
\begin{aligned}
&\|\| C \| \mid= \max _{1 \leq k \leq n} \sum_{p=1}^{n}\left\|\tilde{\alpha}_{k p}\right\| \\
& \leq\left(1+\tilde{L}_{\gamma} T\right)\left(c_{\gamma} \tilde{L}_{1, \gamma}\right)^{2} \Lambda_{n} \max _{1 \leq k \leq n} \\
& \quad \times \int_{t_{k-1}}^{t_{k}}\left|t_{k}-\eta\right|^{1-\gamma} \int_{t_{k-1}}^{\eta}|\eta-s|^{-\gamma}\left|t_{k}-s\right| d s d \eta \\
& \leq\left(1+\tilde{L}_{\gamma} T\right)\left(c_{\gamma} \tilde{L}_{1, \gamma}\right)^{2} \Lambda_{n} \max _{1 \leq k \leq n} \frac{\tau_{k}}{1-\gamma} \\
& \quad \times \int_{t_{k-1}}^{t_{k}}\left|t_{k}-\eta\right|^{1-\gamma}\left|\eta-t_{k-1}\right|^{1-\gamma} d \eta \\
& \leq\left(1+\tilde{L}_{\gamma} T\right)\left(c_{\gamma} \tilde{L}_{1, \gamma}\right)^{2} \Lambda_{n} \max _{1 \leq k \leq n} \frac{\tau_{k}^{2-\gamma}}{1-\gamma} \int_{t_{k-1}}^{t_{k}}\left|t_{k}-\eta\right|^{1-\gamma} d \eta \\
& \leq c(\gamma, T) \Lambda_{n} \tau_{\max }^{4-2 \gamma} \\
& \leq c(\gamma, T) n^{2 \gamma-4} \ln n,
\end{aligned}
$$

where $c(\gamma, T)=c \frac{\left(1+\tilde{L}_{\gamma} T\right)\left(c_{\gamma} \tilde{L}_{1, \gamma}\right)^{2}}{(1-\gamma)(2-\gamma)}, c$ is a constant independent of $n, \gamma$ and 3.20 is proved.

Furthermore, the inequalities (1.4), (1.6), (1.7) imply

$$
\begin{aligned}
\left\|\tilde{S}_{k, k-1}\right\| \leq & e^{-\omega \tau_{k}}+c_{\gamma} \tilde{L}_{1, \gamma}\left(1+\tilde{L}_{\gamma} \tau_{k}\right) \\
& \times \int_{t_{k-1}}^{t_{k}}\left|t_{k}-\eta\right|^{-\gamma}\left|t_{k}-\eta\right| e^{-\omega\left(\eta-t_{k-1}\right)} d \eta \\
\leq & e^{-\omega \tau_{k}}\left[1+\frac{c_{\gamma} \tilde{L}_{1, \gamma}\left(1+\tilde{L}_{\gamma} \tau_{k}\right)}{2-\gamma} \tau_{k}^{2-\gamma}\right]
\end{aligned}
$$

which yields

$$
\left\|\tilde{S}^{-1}\right\| \leq \sum_{p=0}^{n-1} q^{p}=\frac{q^{n}-1}{q-1}
$$

with

$$
q=\left\{e^{-\omega \tau_{\max }}\left[1+\frac{c_{\gamma} \tilde{L}_{1, \gamma}\left(1+\tilde{L}_{\gamma} \tau_{\max }\right)}{2-\gamma} \tau_{\max }^{2-\gamma}\right]\right\} \rightarrow 1
$$

as $\tau_{\max } \rightarrow 0$. This means that there exists a constant $C=C\left(\gamma, c_{\gamma}, \tilde{L}_{\gamma}, \tilde{L}_{1, \gamma}\right)$ such that

$$
\left\|\tilde{S}^{-1}\right\| \leq C n
$$

(it is easy to see that $C \leq 1$ provided that $\left.-\omega+\frac{c_{\gamma} \tilde{L}_{1, \gamma}\left(1+\tilde{L}_{\gamma} \tau_{\max }\right)}{2-\gamma} \tau_{\max }^{1-\gamma} \leq 0\right)$. This estimate together with (3.23) implies (3.21). The proof is complete.

Now, we can go to the first main result of this section.

Theorem 3.4. Let assumptions (1.3)-(1.7) with $\gamma<1$ hold. Then there exists a positive constant $c$ such that the following hold. 
(1) For $n$ large enough it holds that

$$
|\|z\|| \equiv|\|y-u\|| \leq c n^{2 \gamma-3} \ln n E_{n}\left(A_{0}^{\gamma} u\right), \quad \gamma \in[0,1),
$$

where $u$ is the solution of (1.1) and $E_{n}\left(A_{0}^{\gamma} u\right)$ is the best approximation of $A_{0}^{\gamma} u$ by polynomials of degree not greater then $n-1$.

(2) The system of linear algebraic equations

$$
S y=C y+f
$$

from (3.18) with respect to the approximate solution y can be solved by the fixed-point iteration

$y^{(k+1)}=S^{-1} C y^{(k)}+S^{-1}(f-\tilde{f}), \quad k=0,1, \ldots ; \quad y^{(0)}$ arbitrary

converging at least as a geometrical progression with the denominator $q=$ $c(\gamma, T) n^{2 \gamma-3} \ln n<1, \gamma \in[0,1)$ for $n$ large enough.

Proof. From the second equation in (3.18) we get

$$
z=S^{-1} C z+S^{-1} \psi
$$

from which due to Lemma 2.3 and 3.25 wet

$$
|\|z\|| \leq c n|\|\psi\|| \text {. }
$$

Let $\Pi_{n-1}$ be the set of all polynomials in $t$ with vector coefficients of degree less than or equal to $n-1$. Using the Lebesgue inequality (2.33), the last norm can be estimated as

$$
\begin{aligned}
\|\| \psi \|= & \max _{1 \leq k \leq n} \| \int_{t_{k-1}}^{t_{k}} A_{k}^{\gamma} e^{-A_{k}\left(t_{k}-\eta\right)}\left[A_{k}-A(\eta)\right] \\
& \times \int_{t_{k-1}}^{\eta} e^{-A_{k}(\eta-s)}\left[A_{k}-A(s)\right] A_{0}^{-\gamma}\left(A_{0}^{\gamma} u(s)-P_{n-1}\left(s ; A_{0}^{\gamma} u\right)\right) d s d \eta \| \\
\leq & \left(1+\tilde{L}_{\gamma} T\right)\left(c_{\gamma} L_{1, \gamma}\right)^{2} \max _{1 \leq k \leq n} \int_{t_{k-1}}^{t_{k}}\left|t_{k}-\eta\right|^{-\gamma}\left|t_{k}-\eta\right| \\
& \quad \times \int_{t_{k-1}}^{\eta}|\eta-s|^{-\gamma}\left|t_{k}-s\right||| A_{0}^{\gamma} u(\eta)-P_{n-1}\left(s ; A_{0}^{\gamma} u\right) \| d s d \eta \\
\leq & \left(1+\tilde{L}_{\gamma} T\right)\left(c_{\gamma} L_{1, \gamma}\right)^{2}\left(1+\Lambda_{n}\right) E_{n}\left(A_{0}^{\gamma} u\right) \\
\quad & \quad \max _{1 \leq k \leq n}\left\{\int_{t_{k-1}}^{t_{k}}\left|t_{k}-\eta\right|^{-\gamma}\left|t_{k}-\eta\right| \int_{t_{k-1}}^{\eta}|\eta-s|^{-\gamma}\left|t_{k}-s\right| d s d \eta\right\} \\
\leq & c c(\gamma, T) E_{n}\left(A_{0}^{\gamma} u\right) n^{2 \gamma-4} \ln n
\end{aligned}
$$

and taking into account (3.31), we get the first assertion of the theorem.

The second assertion is a simple consequence of (3.28) and (3.21), which completes the proof of the theorem.

Under somewhat stronger assumptions on the operator $A(t)$ one can improve the error estimate for our method in the case $0 \leq \gamma \leq 1$. In order to do it we need the following lemma. 
Lemma 3.5. Let $L_{\nu, n-1}(t)$ be the Lagrange fundamental polynomials related to the Chebyshev interpolation nodes (zeros of the Chebyshev polynomial of the first kind $\left.T_{n}(t)\right)$. Then

$$
\sum_{\nu=1}^{n}\left|L_{\nu, n-1}^{\prime}(t)\right| \leq \frac{1}{\sqrt{1-x^{2}}} \sqrt{2 / 3} n^{3 / 2}
$$

Proof. Let $x \in[-1,1]$ be an arbitrary point and let $\epsilon_{\nu}=\operatorname{sign}\left(L_{\nu, n-1}^{\prime}(x)\right)$. We consider the polynomial of $t$

$$
\rho(t ; x)=\sum_{\nu=1}^{n} \epsilon_{\nu}(x) L_{\nu, n-1}(t)=\sum_{\nu=1}^{n-1} c_{\nu}(x) T_{\nu}(t) .
$$

Since $\rho^{2}(t ; x)$ is the polynomial of degree $2 n-2$, then using the Gauß-Chebyshev quadrature rule and the property $L_{k, n-1}\left(t_{\nu}\right)=\delta_{k, \nu}$ of the fundamental Lagrange polynomials $\left(\delta_{k, \nu}\right.$ is the Kronecker symbol), we get

$$
\begin{aligned}
\int_{-1}^{1} \frac{\rho^{2}(t ; x)}{\sqrt{1-t^{2}}} d t & =\sum_{\nu=0}^{n-1} c_{\nu}^{2}(x) \frac{\pi}{2}=\sum_{\nu=1}^{n} \lambda_{\nu} \rho^{2}\left(t_{\nu} ; x\right) \\
& =\sum_{\nu=1}^{n} \lambda_{\nu} \epsilon_{\nu}^{2}=\sum_{\nu=1}^{n} \lambda_{\nu}=\int_{-1}^{1} \frac{1}{\sqrt{1-t^{2}}} d t=\pi
\end{aligned}
$$

with the quadrature coefficients $\lambda_{\nu}$ which yields

$$
\sum_{\nu=0}^{n-1} c_{\nu}^{2}(x)=2 .
$$

The next estimate

$$
\begin{aligned}
\rho^{\prime}(x) & =\sum_{\nu=1}^{n}\left|L_{\nu, n-1}^{\prime}(x)\right| \leq \sum_{\nu=0}^{n-1}\left|c_{\nu}\right|\left|T_{\nu}^{\prime}(x)\right| \\
& =\sum_{\nu=0}^{n-1}\left|c_{\nu}\right| \frac{\nu}{\sqrt{1-x^{2}}} \leq \frac{1}{\sqrt{1-x^{2}}}\left(\sum_{\nu=0}^{n-1} c_{\nu}^{2}\right)^{1 / 2}\left(\sum_{\nu=1}^{n-1} \nu^{2}\right)^{1 / 2} \\
& \leq \frac{1}{\sqrt{1-x^{2}}}\left(\sum_{\nu=0}^{n-1}\left(c_{\nu}\right)^{2}\right)^{1 / 2} \sqrt{n^{3} / 3}
\end{aligned}
$$

together with (3.36) proves the lemma.

Now we are in the position to prove the following important result of this section.

Lemma 3.6. Let the operator $A(t)$ be strongly continuous differentiable on $[0, T]$ (see [18], Ch. 2, §1, p. 218, 19]), satisfy condition (1.6), and let $A^{\prime}(s) A^{-\gamma}(0)$ be bounded for all $s \in[0, T]$ and $\gamma \in[0,1]$ by a constant $c^{\prime}$. Then for $n$ large enough the following estimates hold true:

$$
\begin{gathered}
|\|C\|| \leq c n^{\gamma-5 / 2}, \quad \gamma \in[0,1], \\
\left|\left\|\tilde{S}^{-1} C\right\|\right| \leq c n^{\gamma-3 / 2}, \quad \gamma \in[0,1]
\end{gathered}
$$

with some positive constant $c$ independent of $n, \gamma$. 
Proof. Opposite to the proof of Lemma 2.3 (see (3.22)), we estimate $\tilde{\alpha}_{k p}$ as

$$
\begin{aligned}
\left\|\tilde{\alpha}_{k p}\right\|= & \left\|A_{k}^{\gamma} \alpha_{k p} A_{p}^{-\gamma}\right\| \\
= & \| \int_{t_{k-1}}^{t_{k}} A_{k}^{\gamma} e^{-A_{k}\left(t_{k}-\eta\right)}\left[A_{k}-A(\eta)\right] \\
& \times A_{k}^{-1} \int_{t_{k-1}}^{\eta} \frac{d e^{-A_{k}(\eta-s)}}{d s}\left[A_{k}-A(s)\right] A_{p}^{-\gamma} L_{p, n-1}(\eta) d \eta \| \\
= & \| \int_{t_{k-1}}^{t_{k}} A_{k}^{\gamma} e^{-A_{k}\left(t_{k}-\eta\right)}\left[A_{k}-A(\eta)\right] A_{k}^{-1}\left\{\left[A_{k}-A(\eta)\right] A_{p}^{-\gamma} L_{p, n-1}(\eta)\right. \\
& -e^{-A_{k}\left(\eta-t_{k-1}\right)}\left[A_{k}-A_{k-1}\right] A_{p}^{-\gamma} L_{p, n-1}\left(t_{k-1}\right) \\
& +\int_{t_{k-1}}^{\eta} e^{-A_{k}(\eta-s)} A^{\prime}(s) A_{p}^{-\gamma} L_{p, n-1}(s) d s \\
& \left.-\int_{t_{k-1}}^{\eta} e^{-A_{k}(\eta-s)}\left[A_{k}-A(s)\right] A_{p}^{-\gamma} L_{p, n-1}^{\prime}(s) d s\right\} d \eta \| \\
\leq & \int_{t_{k-1}}^{t_{k}} c_{\gamma} \tilde{L}_{1,1}\left(t_{k}-\eta\right)^{-\gamma}\left(t_{k}-\eta\right)\left\{\tilde{L}_{1, \gamma}\left(t_{k}-\eta\right)\left(1+\tilde{L}_{\gamma} T\right)\left|L_{p, n-1}(\eta)\right|\right. \\
& +\tilde{L}_{1, \gamma} \tau_{k}\left(1+\tilde{L}_{\gamma} T\right) \delta_{p, k-1}+c^{\prime}\left(1+\tilde{L}_{\gamma} T\right) \int_{t_{k-1}}^{\eta}\left|L_{p, n-1}(s)\right| d s \\
& \left.+\int_{t_{k-1}}^{\eta} \tilde{L}_{1, \gamma}\left(t_{k}-s\right)\left(1+\tilde{L}_{\gamma} T\right)\left|L_{p, n-1}^{\prime}(s)\right| d s\right\} d \eta \\
\leq & \int_{t_{k-1}}^{t_{k}} c_{\gamma} \tilde{L}_{1,1}\left\{\tilde{L}_{1, \gamma}\left(t_{k}-\eta\right)^{2-\gamma}\left|L_{p, n-1}(\eta)\right|\right. \\
& +\tilde{L}_{1, \gamma} \tau_{k}\left(1+\tilde{L}_{\gamma} T\right)\left(t_{k}-\eta\right)^{1-\gamma} \delta_{p, k-1} \\
& +c^{\prime}\left(1+\tilde{L}_{\gamma} T\right)\left(t_{k}-\eta\right)^{1-\gamma} \int_{t_{k-1}}^{\eta}\left|L_{p, n-1}(s)\right| d s \\
& \left.+\tilde{L}_{1, \gamma} \tau_{k}\left(1+\tilde{L}_{\gamma} T\right)\left(t_{k}-\eta\right)^{1-\gamma} \int_{t_{k-1}}^{\eta}\left|L_{p, n-1}^{\prime}(s)\right| d s\right\} d \eta . \\
&
\end{aligned}
$$

Using this inequality together with (3.33), 3.25) and the relations $\arcsin t=\pi / 2-$ $\arccos t, t_{k}=\cos \frac{2 k-1}{2 n} \pi, k=1, \ldots, n$, we arrive at the estimates

$$
\begin{aligned}
\|\| C \| & =\max _{1 \leq k \leq n} \sum_{p=1}^{n}\left\|A_{k}^{\gamma} \alpha_{k, p} A_{p}^{-\gamma}\right\| \\
& \leq M\left\{n^{\gamma-3} \ln n+n^{\gamma-3}+n^{\gamma-3 / 2} \max _{1 \leq k \leq n} \int_{t_{k-1}}^{t_{k}} \frac{1}{\sqrt{1-s^{2}}} d s\right\} \\
& \leq M\left\{n^{\gamma-3} \ln n+n^{\gamma-3}+n^{\gamma-3 / 2} \max _{1 \leq k \leq n}\left(\arcsin t_{k}-\arcsin t_{k-1}\right)\right\} \\
& \leq M n^{\gamma-5 / 2},
\end{aligned}
$$

$$
\text { || } \tilde{S}^{-1} C \| \mid \leq M n^{\gamma-3 / 2}
$$

with a constant $M$ independent of $n$. The proof is complete. 
Remark 3.7. If an operator $A(t)$ is strongly continuous differentiable, then condition (1.6) holds true with $\gamma=1$ and the operator $A(t) A^{-1}(0)$ is uniformly bounded (see [18], Ch. 2, §1, p. 219, [19]).

Now, we can go to the second main result of this section.

Theorem 3.8. Let the assumptions of Lemma 3.6 and conditions (1.3)-(1.7) hold. Then there exists a positive constant $c$ such that the following hold.

(1) For $\gamma \in[0,1)$ and $n$ large enough it holds that

$$
|\|z\|| \equiv|\|y-u\|| \leq c n^{2 \gamma-3} \ln n E_{n}\left(A_{0}^{\gamma} u\right),
$$

where $u$ is the solution of (1.1) and $E_{n}\left(A_{0}^{\gamma} u\right)$ is the best approximation of $A_{0}^{\gamma} u$ by polynomials of degree not greater then $n-1$.

(2) The system of linear algebraic equations (3.28) with respect to the approximate solution $y$ can be solved by the fixed-point iteration

$$
y^{(k+1)}=S^{-1} C y^{(k)}+S^{-1} f, \quad k=0,1, \ldots ; \quad y^{(0)} \text { arbitrary }
$$

converging at least as a geometrical progression with the denominator $q=$ $c(\gamma, T) n^{\gamma-3 / 2}<1$ for $n$ large enough.

Proof. Proceeding analogously as in the proof of Theorem 3.4 and using Lemma 3.6 and (3.25), we get

$$
|\|z\|| \leq c n|\|\psi\|| .
$$

For the norm $|\|\psi\|| \mid$ we have (see (3.32) $)$

$$
|\|\psi\|| \leq c\left(1+\tilde{L}_{\gamma} T\right) E_{n}\left(A_{0}^{\gamma} u\right) n^{2 \gamma-4} \ln n, \quad \gamma \in[0,1)
$$

which together with (3.44) leads to the estimate (3.42) and to the first assertion of the theorem.

The second assertion is a consequence of (3.41). The proof is complete.

Remark 3.9. A simple generalization of Bernstein's theorem (see 20, 22, 21]) to vector-valued functions gives the estimate

$$
E_{n}\left(A_{0}^{\gamma} u\right) \leq \rho_{0}^{-n}
$$

for the value of the best polynomial approximation provided that $A_{0}^{\gamma} u$ can be analytically extended from $[-1,1]$ into an ellipse with the focus at the points $+1,-1$ and with the sum of semi-axes $\rho_{0}>1$.

If $A_{0}^{\gamma} u$ is $p$ times continuously differentiable, then a generalization of Jackson's theorem (see 20, 22, 21]) gives

$$
E_{n}\left(A_{0}^{\gamma} u\right) \leq c_{p} n^{-p} \omega\left(\frac{d^{p} A_{0}^{\gamma} u}{d t^{p}} ; n^{-1}\right)
$$

with the continuity modulus $\omega$.

Further generalizations for Sobolev spaces of vector-valued functions can be proven analogously [3], Ch. 9. Let us define the weighted Banach space of vectorvalued functions $L_{w}^{p}(-1,1), 1 \leq p \leq+\infty$, with the norm

$$
\|u\|_{L_{w}^{p}(-1,1)}=\left(\int_{-1}^{1}\|u(t)\|^{p} w(t) d t\right)^{1 / p}
$$


for $1 \leq p<\infty$ and

$$
\|u\|_{L^{\infty}(-1,1)}=\sup _{t \in(-1,1)}\|u(t)\|
$$

for $p=\infty$. The weighted Sobolev space is defined by

$$
\begin{aligned}
& H_{w}^{m}(-1,1)=\left\{v \in L_{w}^{2}(-1,1): \text { for } 0 \leq k \leq m,\right. \\
& \text { the derivative } \left.\frac{d^{k} v}{d t^{k}} \text { belongs to } L_{w}^{2}(-1,1)\right\}
\end{aligned}
$$

with the norm

$$
\|u\|_{H_{w}^{m}(-1,1)}=\left(\sum_{k=0}^{m}\left\|\frac{d^{k} v}{d t^{k}}\right\|_{L_{w}^{2}(-1,1)}^{2}\right)^{1 / 2} .
$$

Then one gets for the Chebyshev weight $w(t)=\frac{1}{\sqrt{1-t^{2}}}$ (see [3], pp. 295-298), for the polynomial of the best approximation $B_{n}(t)$ and for the interpolation polynomial $P_{n}(t)$ with the Gauss (roots of the Chebyshev polynomial $T_{n+1}(t)$ ), Gauss-Radau (roots of the polynomial $T_{n+1}(t)-\frac{T_{n+1}(-1)}{T_{n}(1)} T_{n}(t)$ ) or the Gauss-Lobatto (roots of the polynomial $p(t)=T_{n+1}(t)+a T_{n}(t)+b T_{n-1}(t)$ with $a, b$ such that $\left.p(-1)=p(1)=0\right)$ nodes

$$
\begin{gathered}
E_{n}(u) \equiv\left\|u-B_{n} u\right\|_{L^{\infty}(-1,1)} \leq c n^{1 / 2-m}\|u\|_{H_{w}^{m}(-1,1)}, \\
\left\|u-B_{n} u\right\|_{L_{w}^{2}(-1,1)} \leq c n^{-m}\|u\|_{H_{w}^{m}(-1,1)}, \\
\left\|u-P_{n} u\right\|_{L_{w}^{2}(-1,1)} \leq c n^{-m}\|u\|_{H_{w}^{m}(-1,1)}, \\
\left\|u^{\prime}-\left(P_{n} u\right)^{\prime}\right\|_{L_{w}^{2}(-1,1)} \leq c n^{2-m}\|u\|_{H_{w}^{m}(-1,1)} .
\end{gathered}
$$

When the function $u$ is analytic in $[-1,1]$ and has a regularity ellipse whose sum of semi-axes equals $e^{\eta_{0}}$, then

$$
\left\|u^{\prime}-\left(P_{n} u\right)^{\prime}\right\|_{L_{w}^{2}(-1,1)} \leq c(\eta) n^{2} e^{-n \eta} \quad \forall \eta \in\left(0, \eta_{0}\right) .
$$

For the Legendre weight $w(t)=1$ one has (see [3], pp. 289-294)

$$
\begin{gathered}
\left\|u-B_{n} u\right\|_{L^{p}(-1,1)} \leq c n^{-m}\|u\|_{H^{m}(-1,1)}, \quad 2<p \leq \infty, \\
\left\|u-B_{n} u\right\|_{H^{l}(-1,1)} \leq c n^{2 l-m+1 / 2}\|u\|_{H^{m}(-1,1)}, \quad 1 \leq l \leq m, \\
\left\|u-P_{n} u\right\|_{H^{l}(-1,1)} \leq c n^{2 l-m+1 / 2}\|u\|_{H^{m}(-1,1)}, \quad 1 \leq l \leq m, \\
\left\|u^{\prime}-\left(P_{n} u\right)^{\prime}\right\|_{L^{2}(-1,1)} \leq c n^{5 / 2-m}\|u\|_{H_{w}^{m}(-1,1)},
\end{gathered}
$$

where the interpolation polynomial $P_{n}(t)$ can be taken with the Gauss (roots of the Legendre polynomial $L_{n+1}(t)$ ), Gauss-Radau (roots of the polynomial $L_{n+1}(t)-$ $\left.\frac{L_{n+1}(-1)}{L_{n}(1)} L_{n}(t)\right)$ or the Gauss-Lobatto (roots of the polynomial $p(t)=L_{n+1}(t)+$ $a L_{n}(t)+b L_{n-1}(t)$ with $a, b$ such that $\left.p(-1)=p(1)=0\right)$ nodes.

Note that the restriction $\gamma \neq 1$ in Theorem 3.8 is only due to the estimate (3.32). Below we show how this restriction can be removed. 
Using (2.30), (3.51), we estimate the norm \|\|$\psi\|\|_{2}$ for $\gamma=1$ as

$$
\begin{aligned}
& |\|\psi\||=\max _{1 \leq k \leq n} \| \int_{t_{k-1}}^{t_{k}} A_{k}^{\gamma} e^{-A_{k}\left(t_{k}-\eta\right)}\left(A_{k}-A(\eta)\right) \\
& \times \int_{t_{k-1}}^{\eta} e^{-A_{k}(\eta-s)}\left(A_{k}-A(s)\right) A_{k}^{-\gamma}\left(A_{k}^{\gamma} A_{0}^{-\gamma}\right)\left(A_{0}^{\gamma} u(s)-P_{n-1}\left(s ; A_{0}^{\gamma} u\right)\right) d s d \eta \| \\
& =\max _{1 \leq k \leq n} \| \int_{t_{k-1}}^{t_{k}} A_{k} e^{-A_{k}\left(t_{k}-\eta\right)}\left(A_{k}-A(\eta)\right) A_{k}^{-1} \\
& \times \int_{t_{k-1}}^{\eta} \frac{d e^{-A_{k}(\eta-s)}}{d s}\left(A_{k}-A(s)\right) A_{k}^{-1}\left(A_{k} A_{0}^{-1}\right)\left(A_{0} u(s)-P_{n-1}\left(s ; A_{0} u\right)\right) d s d \eta \| \\
& =\max _{1 \leq k \leq n} \| \int_{t_{k-1}}^{t_{k}} A_{k} e^{-A_{k}\left(t_{k}-\eta\right)}\left(A_{k}-A(\eta)\right) A_{k}^{-1} \\
& \times\left[\left(A_{k}-A(\eta)\right) A_{k}^{-1}\left(A_{k} A_{0}^{-1}\right)\left(A_{0} u(\eta)-P_{n-1}\left(\eta ; A_{0} u\right)\right)\right. \\
& +\int_{t_{k-1}}^{\eta} e^{-A_{k}(\eta-s)} A^{\prime}(s) A_{k}^{-1}\left(A_{k} A_{0}^{-1}\right)\left(A_{0} u(s)-P_{n-1}\left(s ; A_{0} u\right)\right) d s \\
& -\int_{t_{k-1}}^{\eta} e^{-A_{k}(\eta-s)}\left(A_{k}-A(s)\right) \\
& \left.\times A_{k}^{-1}\left(A_{k} A_{0}^{-1}\right)\left(A_{0} u^{\prime}(s)-\left(P_{n-1}\left(s ; A_{0} u\right)\right)^{\prime}\right) d s\right] d \eta \| \\
& \leq c_{1} \tilde{L}_{1,1} \max _{1 \leq k \leq n} \int_{t_{k-1}}^{t_{k}}\left|t_{k}-\eta\right|^{-1}\left|t_{k}-\eta\right| \\
& \times\left[\left|t_{k}-\eta\right|\left(1+\tilde{L}_{1} T\right) \max _{\eta \in[-1,1]}\left\|\left(A_{0} u(\eta)-P_{n-1}\left(\eta ; A_{0} u\right)\right)\right\|\right. \\
& +c^{\prime}\left(1+\tilde{L}_{1} T\right) \int_{t_{k-1}}^{\eta}\left\|\left(A_{0} u(s)-P_{n-1}\left(s ; A_{0} u\right)\right)\right\| d s \\
& \left.+\left(1+\tilde{L}_{1} T\right) \int_{t_{k-1}}^{\eta}\left|t_{k}-s\right|\left\|\left(A_{0} u^{\prime}(s)-\left(P_{n-1}\left(s ; A_{0} u\right)\right)^{\prime}\right)\right\| d s\right] d \eta \\
& \leq c_{1} \tilde{L}_{1,1} \max _{1 \leq k \leq n}\left[\tau_{k}^{2}\left(1+\tilde{L}_{1} T\right)\left(1+\Lambda_{n}\right) E_{n}\left(A_{0} u\right)\right. \\
& +c^{\prime}\left(1+\tilde{L}_{1} T\right) \tau_{k}^{2}\left(1+\Lambda_{n}\right) E_{n}\left(A_{0} u\right) \\
& \left.+\left(1+\tilde{L}_{1} T\right) \tau_{k}^{2} \int_{t_{k-1}}^{t_{k}}\left\|\left(A_{0} u^{\prime}(s)-\left(P_{n-1}\left(s ; A_{0} u\right)\right)^{\prime}\right)\right\| d s\right] \\
& \leq c_{1} \tilde{L}_{1,1} \max _{1 \leq k \leq n}\left[\tau_{k}^{2}\left(1+\tilde{L}_{1} T\right)\left(1+\Lambda_{n}\right) E_{n}\left(A_{0} u\right)\right. \\
& +c^{\prime}\left(1+\tilde{L}_{1} T\right) \tau_{k}^{2}\left(1+\Lambda_{n}\right) E_{n}\left(A_{0} u\right) \\
& +\left(1+\tilde{L}_{1} T\right) \tau_{k}^{2}\left(\int_{t_{k-1}}^{t_{k}} \frac{1}{\sqrt{1-s^{2}}} d s\right)^{1 / 2} \\
& \left.\times\left(\int_{t_{k-1}}^{t_{k}} \frac{1}{\sqrt{1-s^{2}}}\left\|\left(A_{0} u^{\prime}(s)-\left(P_{n-1}\left(s ; A_{0} u\right)\right)^{\prime}\right)\right\| d s\right)^{1 / 2}\right] \\
& \leq c\left[n^{-2} \ln n E_{n}\left(A_{0} u\right)+n^{-5 / 2}\left\|u^{\prime}-\left(P_{n-1} u\right)^{\prime}\right\|_{L_{w}^{2}(-1,1)}\right] \\
& \leq c\left(n^{-(m+2)} \ln n+n^{-(m+1 / 2)}\right)\|u\|_{H_{w}^{m}(-1,1)} \leq c n^{-(m+1 / 2)}\|u\|_{H_{w}^{m}(-1,1)}
\end{aligned}
$$


provided that the solution $u$ of problem (1.1) belongs to the Sobolev class $H_{w}^{m}(-1,1)$. If $u$ is analytic in $[-1,1]$ and has a regularity ellipse with the sum of the semi-axes equal to $e^{\eta_{0}}>1$, then using (3.52), we get

$$
|\|\psi\|| \leq c\left(\eta_{0}\right) n^{2} e^{-n \eta_{0}} .
$$

Now, Lemma 3.6 together with the last estimates for \|\|$\psi \| \mid$ yields the following third main result of this section.

Theorem 3.10. Let the assumptions of Lemma 3.6 and conditions (1.3)-(1.7) with $\gamma=1$ hold. Then there exists a positive constant $c$ such that the following hold.

(1) For $\gamma=1$ and $n$ large enough we have

$$
|\|z\|| \equiv|\|y-u\|| \leq c n^{-m}\|u\|_{H_{w}^{m}(-1,1)}
$$

provided that the solution $u$ of problem (1.1) belongs to the class $H_{w}^{m}(-1,1)$ with $w(t)=\frac{1}{\sqrt{1-t^{2}}}$.

(2) For $\gamma=1$ and $n$ large enough it holds that

$$
|\|z\|| \equiv|\|y-u\|| \leq c\left(\eta_{0}\right) n^{3 / 2} e^{-n \eta_{0}}
$$

provided that $u$ is analytic in $[-1,1]$ and has a regularity ellipse with the sum of the semi-axes equal to $e^{\eta_{0}}>1$.

(3) The system of linear algebraic equations (3.28) with respect to the approximate solution y can be solved by the fixed-point iteration

$$
y^{(k+1)}=S^{-1} C y^{(k)}+S^{-1} f, \quad k=0,1, \ldots ; \quad y^{(0)} \text { arbitrary }
$$

converging at least as a geometrical progression with the denominator $q=$ $c n^{-1 / 2}<1$ for $n$ large enough.

Remark 3.11. Using estimates (3.53), one can analogously construct a discrete scheme on the Gauss, the Gauss-Radau or the Gauss-Lobatto grids relative to $w(t)=1$ (i.e., connected with the Legendre orthogonal polynomials) and get the corresponding estimates in the $L^{2}(-1,1)$-norm.

\section{Discrete SECOND ORDER PROBlem}

In this section we consider problem (1.2) in a Hilbert space $H$ with the scalar product $(\cdot, \cdot)$ and the corresponding norm $\|\cdot\|=\sqrt{(\cdot, \cdot)}$. Let assumption (1.7) related to $A(t)$ holds. In addition we assume in this section that

$$
A(t)=\sum_{k=0}^{m_{A}} A_{k} t^{k}, \quad f(t)=\sum_{k=0}^{m_{f}} f_{k} t^{k}
$$

and

$$
\left\|A^{\gamma-1 / 2}(0)(A(t)-A(s)) A^{-\gamma}(0)\right\| \leq \tilde{L}_{2, \gamma}|t-s|^{\alpha}, \quad \gamma, \alpha \in[0,1] .
$$

Remark 4.1. Condition (4.2) coincides with (1.6) for $\gamma=1 / 2, \alpha=1$.

Let $\bar{A}(t)$ be a piecewise constant operator defined as in the previous sections. We consider the auxiliary problem

$$
\begin{gathered}
\frac{d^{2} u}{d t^{2}}+\bar{A}(t) u=[\bar{A}(t)-A(t)]+f(t), \\
u(-1)=u_{0}, \quad u^{\prime}(1)=u_{0}^{\prime},
\end{gathered}
$$


from which we get the following relations for the interval $\left[t_{k-1}, t_{k}\right]$ :

$$
\begin{aligned}
u(t) & =\cos \left[\sqrt{A_{k}}\left(t-t_{k-1}\right)\right] u\left(t_{t_{k-1}}\right)+A_{k}^{-1 / 2} \sin \left[\sqrt{A_{k}}\left(t-t_{k-1}\right)\right] u^{\prime}\left(t_{t_{k-1}}\right) \\
& +\int_{t_{k-1}}^{t_{k}} A_{k}^{-1 / 2} \sin \left[\sqrt{A_{k}}(t-\eta)\right]\{[\bar{A}(\eta)-A(\eta)] u(\eta)+f(\eta)\} d \eta, \\
u^{\prime}(t) & =-\sqrt{A_{k}} \sin \left[\sqrt{A_{k}}\left(t-t_{k-1}\right)\right] u\left(t_{t_{k-1}}\right)+\cos \left[\sqrt{A_{k}}\left(t-t_{k-1}\right)\right] u^{\prime}\left(t_{t_{k-1}}\right) \\
& +\int_{t_{k-1}}^{t_{k}} \cos \left[\sqrt{A_{k}}(t-\eta)\right]\{[\bar{A}(\eta)-A(\eta)] u(\eta)+f(\eta)\} d \eta .
\end{aligned}
$$

We chose a grid $\omega_{n}$ of $n$ Chebyshev points as above and substitute in (4.4) the interpolation polynomial (2.7) instead of $u$. Then by collocation we arrive at the following system of linear algebraic equations with respect to the unknowns $y_{k}, y_{k}^{\prime}$ which approximate $u\left(t_{k}\right)$ and $u^{\prime}\left(t_{k}\right)$, respectively:

$$
\begin{aligned}
& y_{k}= \cos \left[\sqrt{A_{k}} \tau_{k}\right] y_{k-1}+A_{k}^{-1 / 2} \sin \left[\sqrt{A_{k}} \tau_{k}\right] y_{k-1}^{\prime} \\
&+\sum_{i=1}^{n} \alpha_{k, i} y_{i}+\phi_{k}^{(1)}, \\
& y_{k}^{\prime}=-\sqrt{A_{k}} \sin \left[\sqrt{A_{k}} \tau_{k}\right] y_{k-1}+\cos \left[\sqrt{A_{k}} \tau_{k}\right] y_{k-1}^{\prime} \\
&+\sum_{i=1}^{n} \beta_{k, i} y_{i}+\phi_{k}^{(2)}, \quad k=1,2, \ldots, n, \\
& y_{0}=u_{0}, \quad y_{0}^{\prime}=u_{0}^{\prime},
\end{aligned}
$$

where

$$
\begin{gathered}
\alpha_{k, i}=\int_{t_{k-1}}^{t_{k}} A_{k}^{-1 / 2} \sin \left[\sqrt{A_{k}}\left(t_{k}-\eta\right)\right]\left[A_{k}-A(\eta)\right] L_{i, n-1}(\eta) d \eta, \\
\beta_{k, i}=\int_{t_{k-1}}^{t_{k}} \cos \left[\sqrt{A_{k}}\left(t_{k}-\eta\right)\right]\left[A_{k}-A(\eta)\right] L_{i, n-1}(\eta) d \eta, \\
\phi_{k}^{(1)}=\int_{t_{k-1}}^{t_{k}} A_{k}^{-1 / 2} \sin \left[\sqrt{A_{k}}\left(t_{k}-\eta\right)\right] f(\eta) d \eta, \\
\phi_{k}^{(2)}=\int_{t_{k-1}}^{t_{k}} \cos \left[\sqrt{A_{k}}\left(t_{k}-\eta\right)\right] f(\eta) d \eta, \quad k=1,2, \ldots, n .
\end{gathered}
$$

The errors $z_{k}=u\left(t_{k}\right)-y_{k}, z_{k}^{\prime}=u^{\prime}\left(t_{k}\right)-y_{k}^{\prime}$ satisfy the equations

$$
\begin{aligned}
& z_{k}= \cos \left[\sqrt{A_{k}} \tau_{k}\right] z_{k-1}+A_{k}^{-1 / 2} \sin \left[\sqrt{A_{k}} \tau_{k}\right] z_{k-1}^{\prime} \\
&+\sum_{i=1}^{n} \alpha_{k, i} z_{i}+\psi_{k}^{(1)}, \\
& z_{k}^{\prime}=-\sqrt{A_{k}} \sin \left[\sqrt{A_{k}} \tau_{k}\right] z_{k-1}+\cos \left[\sqrt{A_{k}} \tau_{k}\right] z_{k-1}^{\prime} \\
&+\sum_{i=1}^{n} \beta_{k, i} z_{i}+\psi_{k}^{(2)}, \quad k=1,2, \ldots, n, \\
& z_{0}=0, \quad z_{0}^{\prime}=0,
\end{aligned}
$$


where

$$
\begin{gathered}
\psi_{k}^{(1)}=\int_{t_{k-1}}^{t_{k}} A_{k}^{-1 / 2} \sin \left[\sqrt{A_{k}}\left(t_{k}-\eta\right)\right]\left[A_{k}-A(\eta)\right]\left[u(\eta)-P_{n-1}(\eta ; u)\right] d \eta, \\
\psi_{k}^{(2)}=\int_{t_{k-1}}^{t_{k}} \cos \left[\sqrt{A_{k}}\left(t_{k}-\eta\right)\right]\left[A_{k}-A(\eta)\right]\left[u(\eta)-P_{n-1}(\eta ; u)\right] \\
d \eta, k=1,2, \ldots, n .
\end{gathered}
$$

Let us denote $\tilde{y}_{k}=A_{k}^{\gamma} y_{k}, \tilde{y}_{k}^{\prime}=A_{k}^{\gamma-1 / 2} y_{k}^{\prime}, \tilde{z}_{k}=A_{k}^{\gamma} z_{k}, \tilde{z}_{k}^{\prime}=A_{k}^{\gamma-1 / 2} z_{k}^{\prime}$ and rewrite (4.7) in the form

$$
\begin{aligned}
& \tilde{z}_{k}= \cos \left[\sqrt{A_{k}} \tau_{k}\right]\left(A_{k}^{\gamma} A_{k-1}^{-\gamma}\right) \tilde{z}_{k-1} \\
&+\sin \left[\sqrt{A_{k}} \tau_{k}\right]\left(A_{k}^{\gamma-1 / 2} A_{k-1}^{-(\gamma-1 / 2)}\right) \tilde{z}_{k-1}^{\prime}+\sum_{i=1}^{n} \tilde{\alpha}_{k, i} \tilde{z}_{i}+\tilde{\psi}_{k}^{(1)}, \\
& \tilde{z}_{k}^{\prime}=-\sin \left[\sqrt{A_{k}} \tau_{k}\right]\left(A_{k}^{\gamma} A_{k-1}^{-\gamma}\right) \tilde{z}_{k-1} \\
&+\cos \left[\sqrt{A_{k}} \tau_{k}\right]\left(A_{k}^{\gamma-1 / 2} A_{k-1}^{-(\gamma-1 / 2)}\right) \tilde{z}_{k-1}^{\prime}+\sum_{i=1}^{n} \tilde{\beta}_{k, i} \tilde{z}_{i}+\tilde{\psi}_{k}^{(2)}, \quad k=1,2, \ldots, n, \\
& z_{0}=0, \quad \tilde{z}_{0}^{\prime}=0,
\end{aligned}
$$

where

$$
\begin{aligned}
\tilde{\alpha}_{k, i} & =A_{k}^{\gamma} \alpha_{k, i} A_{i}^{-\gamma} \\
& =\int_{t_{k-1}}^{t_{k}} A_{k}^{\gamma-1 / 2} \sin \left[\sqrt{A_{k}}\left(t_{k}-\eta\right)\right]\left[A_{k}-A(\eta)\right] A_{i}^{-\gamma} L_{i, n-1}(\eta) d \eta, \\
\tilde{\beta}_{k, i} & =A_{k}^{\gamma-1 / 2} \beta_{k, i} A_{i}^{-\gamma} \\
& =\int_{t_{k-1}}^{t_{k}} A_{k}^{\gamma-1 / 2} \cos \left[\sqrt{A_{k}}\left(t_{k}-\eta\right)\right]\left[A_{k}-A(\eta)\right] A_{i}^{-\gamma} L_{i, n-1}(\eta) d \eta, \\
\tilde{\psi}_{k}^{(1)} & =A_{k}^{\gamma} \phi_{k}^{(1)} \\
& =\int_{t_{k-1}}^{t_{k}} A_{k}^{\gamma-1 / 2} \sin \left[\sqrt{A_{k}}\left(t_{k}-\eta\right)\right] A_{k}^{-\gamma}\left[A_{k}^{\gamma}\left(u(\eta)-P_{n-1}(\eta ; u)\right)\right] d \eta, \\
\tilde{\psi}_{k}^{(2)} & =A_{k}^{\gamma-1 / 2} \phi_{k}^{(2)} \\
& =\int_{t_{k-1}}^{t_{k}} A_{k}^{\gamma-1 / 2} \cos \left[\sqrt{A_{k}}\left(t_{k}-\eta\right)\right] A_{k}^{-\gamma}\left[A_{k}^{\gamma}\left(u(\eta)-P_{n-1}(\eta ; u)\right)\right] d \eta .
\end{aligned}
$$

We introduce the $2 \times 2$-block matrices

$$
\begin{gathered}
E=\left(\begin{array}{cc}
I & 0 \\
0 & I
\end{array}\right), \quad B_{k}\left(t_{k}-\eta\right)=\left(\begin{array}{cc}
\cos \left(\sqrt{A_{k}}\right)\left(t_{k}-\eta\right) & \sin \left(\sqrt{A_{k}}\right)\left(t_{k}-\eta\right) \\
-\sin \left(\sqrt{A_{k}}\right)\left(t_{k}-\eta\right) & \cos \left(\sqrt{A_{k}}\right)\left(t_{k}-\eta\right)
\end{array}\right), \\
D_{k}=\left(\begin{array}{cc}
A_{k}^{\gamma} A_{k-1}^{-\gamma} & 0 \\
0 & A_{k}^{\gamma-1 / 2} A_{k-1}^{-(\gamma-1 / 2)}
\end{array}\right), \quad F_{i}(\eta)=\left(\begin{array}{cc}
A_{k}^{\gamma-1 / 2}\left[A_{i}-A(\eta)\right] A_{i}^{-\gamma} & 0 \\
0 & 0
\end{array}\right),
\end{gathered}
$$


and

$$
\begin{aligned}
& S=\left(\begin{array}{ccccccc}
E & 0 & 0 & \cdots & 0 & 0 & 0 \\
-B_{2} D_{2} & E & 0 & \cdots & 0 & 0 & 0 \\
0 & -B_{3} D_{3} & E & \cdots & 0 & 0 & 0 \\
\cdot & \cdot & \cdot & \cdots & \cdot & \cdot & \cdot \\
0 & 0 & 0 & \cdots & -B_{n-1} D_{n-1} & E & 0 \\
0 & 0 & 0 & \cdots & 0 & -B_{n} D_{n} & E
\end{array}\right), \\
& C \equiv\left\{c_{i, j}\right\}_{i, j=1}^{n}=\left(\begin{array}{ccccccc}
\tilde{\alpha}_{11} & 0 & \tilde{\alpha}_{12} & 0 & \cdots & \tilde{\alpha}_{1 n} & 0 \\
\tilde{\beta}_{11} & 0 & \tilde{\beta}_{12} & 0 & \cdots & \tilde{\beta}_{1 n} & 0 \\
\cdot & \cdot & \cdot & \cdot & \cdots & \cdot & \cdot \\
\tilde{\alpha}_{n 1} & 0 & \tilde{\alpha}_{n 2} & 0 & \cdots & \tilde{\alpha}_{n n} & 0 \\
\tilde{\beta}_{n 1} & 0 & \tilde{\beta}_{n 2} & 0 & \cdots & \tilde{\beta}_{n n} & 0
\end{array}\right)
\end{aligned}
$$

with $B_{k}=B_{k}\left(t_{k}-t_{k-1}\right)=B_{k}\left(\tau_{k}\right)$ and the $2 \times 2$-operator blocks

$$
c_{i, j}=\left(\begin{array}{cc}
\tilde{\alpha}_{i, j} & 0 \\
\tilde{\beta}_{i, j} & 0
\end{array}\right) .
$$

These blocks can also be represented as

$$
c_{i, j}=\int_{t_{i-1}}^{t_{i}} L_{j, n}(\eta) D_{i}^{*} B_{i}^{*}\left(t_{i}-\eta\right) F_{i}(\eta) d \eta .
$$

Using the integral representation of functions of self-adjoint operators by the corresponding spectral family, one can easily show that

$$
\begin{aligned}
B_{k} B_{k}^{*} & =B_{k}^{*} B_{k}=E \\
B_{k}\left(t_{k}-\eta\right) B_{k}\left(t_{k}-\eta\right)^{*} & =B_{k}\left(t_{k}-\eta\right)^{*} B_{k}\left(t_{k}-\eta\right)=E .
\end{aligned}
$$

Analogously, as in the previous section we get

$$
S^{-1} \equiv\left\{s_{i, k}^{(-1)}\right\}_{i, k=1}^{n}=\left(\begin{array}{ccccc}
E & 0 & 0 & \cdots & 0 \\
B_{2} D_{2} & E & 0 & \cdots & 0 \\
B_{3} D_{3} B_{2} D_{2} & B_{3} D_{3} & E & \cdots & 0 \\
\cdot & \cdot & \cdot & \cdots & \cdot \\
B_{n} D_{n} \cdots B_{2} D_{2} & B_{n} D_{n} \cdots B_{3} D_{3} & B_{n} D_{n} \cdots B_{4} D_{4} & \cdots & E
\end{array}\right)
$$

with $2 \times 2$-operator block-elements $s_{i, k}^{(-1)}$. We introduce the vectors

$$
\begin{gathered}
z=\left(w_{1}, w_{2}, \ldots, w_{n}\right)=\left(\tilde{z_{1}}, z_{1}^{\prime}, \ldots, \tilde{z_{n}}, z_{n}^{\prime}\right), \\
w_{i}=\left(z_{i}, z_{i}^{\prime}\right), z_{i}, z_{i}^{\prime} \in H, \\
\psi=\left(\Psi_{1}, \Psi_{2}, \ldots, \Psi_{n}\right)=\left(\tilde{\psi}_{1}^{(1)}, \psi_{1}^{(2)}, \ldots, \tilde{\psi}_{n}^{(1)}, \psi_{n}^{(2)}\right), \\
\Psi_{i}=\left(\tilde{\psi}_{i}^{(i)}, \psi_{i}^{(2)}\right), \tilde{\psi}_{i}^{(i)}, \psi_{i}^{(2)} \in H .
\end{gathered}
$$

Then equations (4.9) can be written in block matrix form as

$$
z=S^{-1} C z+S^{-1} \psi \text {. }
$$

Note that the block vectors $\Psi_{i}$ can be written as

$$
\Psi_{i}=\int_{t_{i-1}}^{t_{i}} \tilde{B}_{i}^{*}\left(t_{i}-\eta\right) D_{\psi}(\eta) d \eta
$$


with the block vectors

$$
D_{\psi}(\eta)=\left(\begin{array}{c}
0 \\
A^{\gamma-1 / 2}\left[A_{i}-A(\eta)\right] A_{i}^{-\gamma}\left[A_{i}^{\gamma}\left(u(\eta)-P_{n}(\eta ; u)\right)\right]
\end{array}\right) .
$$

The blocks $E, B_{i}$ act in the space of two-dimensional block vectors $v=\left(v_{1}, v_{2}\right)$, $v_{1}, v_{2} \in H$. In this space we define the new scalar product by

$$
((u, v))=\left(u_{1}, v_{1}\right)+\left(u_{2}, v_{2}\right),
$$

the corresponding block-vector norm by

$$
\|v\|_{b}=\sqrt{((v, v))}=\left(\left\|v_{1}\right\|^{2}+\left\|v_{2}\right\|^{2}\right)^{1 / 2}
$$

and the consistent norm for a block operator matrix $G=\left(\begin{array}{ll}g_{11} & g_{12} \\ g_{21} & g_{22}\end{array}\right)$ by

$$
\mid\|G\|_{b}=\sup _{y \neq 0} \frac{\sqrt{((G v, G v))}}{|\|v\||} .
$$

In the space of $n$-dimensional block vectors we define the block-vector norm by

$$
|\|y\||=\max _{1 \leq k \leq n}\left|\left\|v_{k}\right\|\right|_{b}
$$

and the consistent matrix norm

$$
|\|C\|| \equiv\left|\left\|\left\{c_{i, j}\right\}_{i, j=1}^{n}\right\|\right|=\sup _{y \neq 0} \frac{|\|C y\||}{|\|y\||}=\max _{1 \leq k \leq n} \sum_{p=1}^{n}\left|\left\|c_{k p}\right\|\right|_{b} .
$$

It is easy to see that

$$
\begin{aligned}
\left|\left\|B_{i}\right\|\right|_{b} & =\left|\left\|B_{i}^{*}\right\|\right|_{b}=\sup _{v \neq 0} \frac{\left|\left\|B_{i} v\right\|\right|}{|\|v\||}=\sup _{v \neq 0} \frac{\sqrt{\left(\left(B_{i} v, B_{i} v\right)\right)}}{|\|v\||} \\
& =\sup _{v \neq 0} \frac{\sqrt{\left(\left(B_{i} B_{i}^{*} v, v\right)\right)}}{|\|v\||}=1
\end{aligned}
$$

and due to 1.7

$$
\begin{aligned}
&\left|\left\|D_{k}\right\|\right|_{b}=\sup _{v \neq 0} \frac{\left|\left\|D_{k} v\right\|\right|}{|\|v\||}=\sup _{v \neq 0} \frac{\sqrt{\left(\left(D_{k} v, D_{k} v\right)\right)}}{|\|v\||} \\
&=\sup _{v \neq 0} \frac{\sqrt{\left(\left(D_{k} D_{i} k^{*} v, v\right)\right)}}{|\|v\||} \leq c_{D}, \\
& \mid\left\|D_{k}^{*}\right\| \|_{b} \leq c_{D}
\end{aligned}
$$

with

$$
c_{D}=\sqrt{\left(1+\tilde{L}_{\gamma} T\right)^{2}+\left(1+\tilde{L}_{\gamma-1 / 2} T\right)^{2}} .
$$

Let us estimate $\left|\left\|S^{-1}\right\|\right|$. Due to (4.24), (4.25), (4.23) and (4.14) we have

$$
\left|\left\|S^{-1}\right\|\right| \leq c_{D} n .
$$


Using assumption (4.2) and (4.13), (4.26), we get

$$
\begin{aligned}
&\left|\left\|c_{i, j}\right\|\right|_{b} \leq \int_{t_{i-1}}^{t_{i}}\left|\left\|D_{i}^{*}\right\|\right|||\left|B_{i}\left(t_{i}-\eta\right)\left\||||| F_{i}(\eta)\right\|\right|\left|L_{j, n}(\eta)\right| d \eta \\
& \leq c_{D} \tilde{L}_{2, \gamma} \tau_{\max }^{\alpha} \int_{t_{i-1}}^{t_{i}}\left|L_{j, n-1}(\eta)\right|^{2} d \eta \\
&|\|C\|| \leq \max _{1 \leq k \leq n} \sum_{p=1}^{n}\left|\left\|c_{k, p}\right\|\right|_{b} \\
& \leq c \tau_{\max }^{\alpha}\left(\int_{t_{k-1}}^{t_{k}} \sum_{j=1}^{n}\left|L_{j, n}(\eta)\right| d \eta\right) \leq c \Lambda_{n} \tau_{\max }^{1+\alpha} \leq c n^{-1-\alpha} \ln n, \\
&||\left|S^{-1} C \|\right| \leq n^{-\alpha} \ln n,
\end{aligned}
$$

with some positive constant $c$ independent of $n$.

Now we are in a position to prove the main result of this section.

Theorem 4.2. Let assumptions (1.3)-(4.1), (4.2), (1.7) hold. Then there exists a positive constant $c$ such that the following hold.

(1) For $n$ large enough it holds that

$$
|\|z\|| \equiv|\|y-u\|| \leq c n^{-\alpha} \ln n E_{n}\left(A_{0}^{\gamma} u\right)
$$

where $u$ is the solution of (1.2) and $E_{n}\left(A_{0}^{\gamma} u\right)$ is the best approximation of $A_{0}^{\gamma} u$ by polynomials of degree not greater then $n-1$.

(2) The system of linear algebraic equations

$$
S y=C y+f
$$

with respect to the approximate solution y can be solved by the fixed-point iteration

$$
y^{(k+1)}=S^{-1} C y^{(k)}+S^{-1} f, \quad k=0,1, \ldots ; \quad y^{(0)} \text { arbitrary }
$$

which converges as a geometric progression with the denominator $q=$ $c n^{-\alpha} \ln n<1$ for $n$ large enough.

Proof. Due to (4.16), (4.26) for $\tau_{\max }$ small enough (or for $n$ large enough) there exists a bounded norm $\left|\left\|\left(E-S^{-1} S\right)^{-1}\right\|\right|$ and we get

$$
|\|z\|| \leq c n|\|\psi\|| \text {. }
$$

It remains to estimate $\mid\|\psi\| \|$. Using (4.20), (4.17), (4.18) and (4.13), we have

$$
\begin{aligned}
\|\| \psi \| & =\max _{1 \leq k \leq n}\left|\left\|\Psi_{k}\right\|\right|_{b} \\
& =\max _{1 \leq k \leq n}\left|\left\|\int_{t_{k-1}}^{t_{k}} \tilde{B}_{k}^{*}\left(t_{k}-\eta\right) D_{\psi}(\eta) d \eta\right\|\right| \\
& \leq \max _{1 \leq k \leq n} \int_{t_{k-1}}^{t_{k}}\left|t_{k}-\eta\right|^{\alpha}\left\|A_{k}^{\gamma} A_{0}^{-\gamma}\right\|\left\|\left[A_{0}^{\gamma}\left(u(\eta)-P_{n}(\eta ; u)\right)\right]\right\| d \eta \\
& \leq\left(1+\tilde{L}_{\gamma} T\right) \tau_{\max }^{1+\alpha}\left(1+\Lambda_{n}\right) E_{n}\left(A_{0}^{\gamma} u\right) \\
& \leq c\left(1+\tilde{L}_{\gamma} T\right) n^{-1-\alpha} \ln n E_{n}\left(A_{0}^{\gamma} u\right) .
\end{aligned}
$$

This inequality together with (4.31) completes the proof of the first assertion. The second one can be proved analogously as in Theorem 3.4 . 
Remark 4.3. We arrive at an exponential accuracy for piecewise analytical solutions if we apply the methods described above successively on each subinterval of the analyticity.

\section{ACKNOWLEDGEMENT}

The authors are grateful for financial support given in part by Deutsche Forschungsgemeinschaft (DFG).

\section{REFERENCES}

[1] K.I. Babenko: Fundamentals of Numerical Analysis, Nauka, Moscow, 1986 (in Russian). MR 0889669 (88g:65001)

[2] H. Bateman and A. Erdélyi: Higher transcendental functions, vol. 2, McGraw-Hill Book Company, Inc., 1953. MR0058756 (15:419i)

[3] C. Canuto, M.Y. Hussaini, A. Quarteroni and T.A. Zang: Spectral Methods in Fluid Dynamics, Springer-Verlag 1988. MR0917480 (89m:76004)

[4] P.G. Ciarlet and J.-L. Lions (eds.): Handbook of Numerical Analysis, Vol. 1, North-Holland, Amsterdam, 1990. MR.1039323 (91f:65001)

[5] R. Dautray and J.-L. Lions: Mathematical Analysis and Numerical Methods for Science and Technology, Vol. 5, Evolution Problems I, Springer-Verlag 1992. MR1156075 (92k:00006)

[6] H. Fattorini: Second Order Linear Differential Equations in Banach Spaces, North-Holland, 1985, pp. 165-237. MR0797071 (87b:34001)

[7] H. Fujita, N. Saito and T. Suzuki: Operator Theory and Numerical Methods, Elsevier, 2001. MR $1854280(2002 \mathrm{i}: 65111)$

[8] I.P. Gavrilyuk: Strongly P-positive operators and explicit representation of the solutions of initial value problems for second order differential equations in Banach space. Journ. of Math. Analysis and Appl. 236 (1999), 327-349. MF 1704587 (2001j:34072)

[9] I.P. Gavrilyuk, W. Hackbusch and B.N. Khoromskij: $\mathcal{H}$-Matrix Approximation for the Operator Exponential with Applications. Numer. Math. 92 (2002) 1, 83-111. MR1917366 (2003g:65061)

[10] I.P. Gavrilyuk, W. Hackbusch and B.N. Khoromskij: $\mathcal{H}$-Matrix approximation for elliptic solution operators in cylinder domains. East-West J. of Num. Math., vol. 9(2001), No. 1, pp. 25-59. MR1839197|(2002e:65064)

[11] I.P. Gavrilyuk, W. Hackbusch, and B. Khoromskij: Data-Sparse Approximation to a Class of Operator-Valued Functions, Math. Comp., posted on August 23, 2004, PII S00255718(04)01703-X (to appear in print).

[12] I.P. Gavrilyuk, W. Hackbusch, B. Khoromskij: Data-Sparse Approximation to the OperatorValued Functions of Elliptic Operators, Math. Comp,. 73 (2004) 1297-1324.

[13] I.P. Gavrilyuk and V.L. Makarov: Exponentially convergent parallel discretization methods for the first order evolution equations, Computational Methods in Applied Mathematics, Vol. 1, No. 4, 2001, pp. 333- 355. MR1892950 (2003f:65174)

[14] I.P. Gavrilyuk and V.L. Makarov: Representation and approximation of the solution of an initial value problem for a first order differential eqation in Banach space. Z. Anal. Anwend., 15 (1996), 495-527. MF 1394440 (97h:65076)

[15] I.P. Gavrilyuk, V.L. Makarov and V.L. Rjabichev: A parallel method of high accuracy for the first order evolution equation in Hilbert and Banach space. Computational Methods in Applied Mathematics, Vol. 3, No. 1, 2003, pp. 86-115. MR2002259 (2004g:65079)

[16] W. Hackbusch: A sparse matrix arithmetic based on $\mathcal{H}$-matrices. Part I: Introduction to $\mathcal{H}$-matrices. Computing 62 (1999), 89-108. MR1694265 (2000c:65039)

[17] W. Hackbusch and B. N. Khoromskij: A sparse $\mathcal{H}$-matrix arithmetic. Part II: Application to multi-dimensional problems. Computing 64 (2000), 21-47. MR,1755846 (2001i:65053)

[18] S.G. Krein: Linear Differential Operators in Banach Spaces, Moscow: Nauka, 1967 (in Russian). MR0247239 (40:508)

[19] S.G. Krein: Linear Differential Operators in Banach Spaces (Transl. Amer. Math. Soc.: vol. 29). New York: Amer. Math. Soc., 1971. MR0342804 (49:7548)

[20] I.P. Natanson: Constructive function theory, Moscow, 1949. MR0034464 (11:591c) 
[21] I.P. Natanson: Constructive function theory. V. 1: Uniform approximation, translated from Russian by N. Obolensky. V. 2: Approximation in mean, translated from Russian by John R. Schulenberger. V. 3: Interpolation and approximation quadratures, translated from Russian by John R. Schulenberger, Ungar, New York, 1964, 1965, 1965. MR0196340 (33:4529a). MR 0196341 (33:4529b): MR0196342(33:4529c)

[22] I.P. Natanson: Konstruktive Funktionentheorie, Akademie-Verlag, Berlin, 1955. MR0069915 (16:1100d)

[23] A. Pazy: Semigroups of linear operator and applications to partial differential equations, Springer Verlag, 1983. MR0710486 (85g:47061)

[24] A.A. Samarskii, I.P. Gavrilyuk and V.L. Makarov: Stability and regularization of three-level difference schemes with unbounded operator coefficients in Banach spaces, SIAM Journal on Numerical Analysis, v. 39, No. 2, 2001, pp. 708-723. MR1860256 (2002h:35163)

[25] A.A. Samarskii, P.N. Vabischevich and P.P. Matus: Difference Schemes with Operator Factors, Kluwer Academic Publishers, Boston/Dordrecht/London, 2002. MR1950844 (2003k:65095)

[26] F. Stenger: Numerical methods based on Sinc and analytic functions. Springer Verlag, 1993. MR, $1226236(94 \mathrm{k}: 65003)$

[27] F. Stenger, A. Naghsh-Nilchi, J. Niebsch, and R. Ramlau: Sampling Methods for Approximate Solution of PDE, in "Inverse Problems and Image Analysis" (eds. Z. Nashed and O. Scherzer), AMS, 2002, pp. 199-250. MR1940998 (2003m:35013)

[28] G. Szegö: Orthogonal Polynomials. American Mathematical Society, New York, 1959. MR0106295 (21:5029)

[29] G. Szegö: Orthogonal Polynomials (with an Introduction and a Complement by J.L. Geronimus). State Publishing House of Physical and Mathematical Literature, Moscow, 1962.

[30] J.A. Goldstein: Semigroups of Linear Operators and Applications, Oxford University Press, New York, and Clarendon Press, Oxford, 1985. MR.0790497 (87c:47056)

Berufsakademie Thüringen, Am Wartenberg 2, D-99817 Eisenach, Germany

E-mail address: ipg@ba-eisenach.de

National Academy of Sciences of Ukraine, Institute of Mathematics, TereschenKIVSKA 3, 01601 KiEV, UKRAINE

E-mail address: makarov@imath.kiev.ua 